\title{
A Lattice Model on the Rate of DNA Hybridization
}

\section{Rajamanickam Murugan*}

Department of Biotechnology, Indian Institute of Technology Madras, Chennai, Tamil Nadu, India. *Email: rmurugan@gmail.com 
Lattice model on DNA hybridization

\begin{abstract}
We develop a lattice model on the rate of hybridization of the complementary single-stranded DNAs (c-ssDNAs). Upon translational diffusion mediated collisions, c-ssDNAs interpenetrate each other to form correct (cc), incorrect (icc) and trap-correct contacts (tcc) inside the reaction volume. Correct contacts are those with exact registry matches which leads to nucleation and zipping. Incorrect contacts are the mismatch contacts which are less stable compared to tcc which can occur in the repetitive c-ssDNAs. Although tcc possess registry match within the repeating sequences, they are incorrect contacts in the view of the whole c-ssDNAs. The nucleation rate $\left(k_{N}\right)$ is directly proportional to the collision rate and the average number of correct-contacts $\left(\left\langle n_{c c}\right\rangle\right)$ formed when both the c-ssDNAs interpenetrate each other. Detailed lattice model simulations suggest that $\left\langle n_{c c}\right\rangle \propto L / V$ where $L$ is the length of cssDNAs and $V$ is the reaction volume. Further numerical analysis revealed the scaling for the average radius of gyration of c-ssDNAs $\left(\mathrm{R}_{\mathrm{g}}\right)$ with their length as $R_{g} \propto \sqrt{L}$. Since the reaction space will be approximately a sphere with radius equals to $2 R_{g}$ and $V \propto L^{3 / 2}$, one obtains $k_{N} \propto \frac{1}{\sqrt{L}}$. When c-ssDNAs are nonrepetitive, then the overall renaturation rate becomes as $k_{R} \propto k_{N} L$ and one finally obtains $k_{R} \propto \sqrt{L}$ in line with the experimental observations. When c-ssDNAs are repetitive with a complexity of $c$, then earlier models suggested the scaling $k_{R} \propto$ $\frac{\sqrt{L}}{c}$ which breaks down at $c=L$. This clearly suggested the existence of at least two different pathways of renaturation in case of repetitive c-ssDNAs viz. via incorrect contacts and trap correct contacts. The trap correct contacts can lead to the formation of partial duplexes which can keep the complementary strands in the close vicinity for a prolonged timescale. This is essential for the extended 1D slithering, inchworm movements and internal displacement mechanisms which can accelerate the searching for the correct contacts. Clearly, the extent of slithering dynamics will be inversely proportional to the complexity. When the complexity is close to the length of c-ssDNAs, then the pathway via incorrect contacts will dominate. When the complexity is much lesser than the length of c-ssDNA, then pathway via trap correct contacts would be the dominating one.
\end{abstract}

\title{
KEY WORDS
}

DNA renaturation, lattice model, DNA hybridization kinetics, correct-contacts, excluded volume effects 
Lattice model on DNA hybridization

\section{Introduction}

The unwind-rewind property of the double stranded helical structure of DNA is critical for its biological function ${ }^{1,2}$. Heating the solution containing the double stranded DNA (dsDNA) melts the hydrogen bonding network across the nitrogen bases and the hydrophobic forces arising out of base-stacking at the core of dsDNA ${ }^{1}$. The process of melting of dsDNA into the corresponding complementary single strands is called as denaturation ${ }^{2}$. Upon cooling the solution well below the melting temperature $\left(T_{m}\right)$, these single strands of DNA (ssDNA) reunite spontaneously to form the original dsDNA. This process is known as DNA renaturation or hybridization ${ }^{2-4}$. Core molecular biological processes such as transcription, translation and replication and several in vitro laboratory techniques are based on the reversible unwindingrewinding property of the dsDNA ${ }^{5}$. Clear understanding on the dynamics and mechanism of DNA hybridization in solution is important in recombination techniques, design of primers for polymerase chain reaction (PCR), design of oligonucleotide probes for microarray chips, design and construction of versatile nano-structures over single stranded DNA scaffolds using DNA origami method ${ }^{6,7}$, various membrane blotting and other related DNA fingerprinting technologies ${ }^{5}$. Several models describing the process of renaturation in aqueous condition have been developed and experimentally tested 2, 8-17. Detailed understanding of the mechanism of hybridization of ssDNAs at the microscopic level is one of the contemporary topics in the biological physics field.

Hybridization of the complementary ssDNAs (c-ssDNAs) was initially modelled as a one-step diffusion controlled bimolecular collision process as in Scheme I of Fig. 1A ${ }^{4,18-20}$. Although this model was enough to fit the experimental kinetic data $8,14,17,19$, it could not explain several other polymer physics related scaling relationships. Particularly, Scheme I predicted a linear scaling of the bimolecular collision rate with respect to the length of c-ssDNAs. Whereas, the experimental data revealed approximately a square root type scaling ${ }^{8,14}$. Further experiments revealed an inverse scaling of the bimolecular collision rate with the sequence complexity of ssDNA. Wetmur and Davidson ${ }^{8}$ suggested a detailed two-step model that comprised of nucleation and zipping as in Scheme II of Fig. 1A. In their model, the overall renaturation rate was directly proportional to the product of nucleation rate and length of cssDNAs and inversely proportional to the sequence complexity. Here, the nucleation rate scales with the length of c-ssDNA in an inverse square root manner. As a result, the overall renaturation rate scales with the length of SSDNA in a square root manner. They further argued that the renaturation was not a diffusion controlled process, and the inverse scaling of the nucleation rate with the length of c-ssDNA must be due to either the excluded volume effects associated with the intra-strand dynamics or steric hindrance associated with the interpenetration of c-ssDNAs that is essential for the nucleation step ${ }^{8}$. However, this argument did not explain the inverse scaling of the renaturation rate with the viscosity of the medium. To overcome this issue, they further assumed a diffusion mediated growth of the nucleus with a rate that is inversely related with the viscosity coefficient of the medium. This assumption ensured the inverse scaling of the overall renaturation rate with the viscosity of the reaction medium.

The c-ssDNAs are random coils with average radius of gyration $R_{g}$ (Fig. 1B). At coarse grained level, one can think both the complementary strands as loosely packed spherical clusters of nucleotide monomers with radius $R_{g}$ performing translational diffusion. The base clusters of c-ssDNAs interpenetrate each other upon collision and subsequently nucleation occurs inside 
the reaction volume. Clearly, there are two distinct dynamical regimes in the process of renaturation viz. 1) translational diffusion mediated collision of base clusters of c-ssDNAs and 2 ) their interpenetration. Translational diffusion brings both the strands within the reaction radius. Reaction radius will be the sum of the radius of gyration of c-ssDNAs. Subsequently, these c-ssDNA base clusters interpenetrate each other within the reaction volume to achieve the nucleation and zipping. Therefore, the translational diffusion component cannot be ignored here. Further, the expression for the reaction rate corresponding to the two-step model (Eq. 3 in Ref. ${ }^{8}$ ) will be inconsistent whenever the sequence complexity has the same magnitude as that of the length of c-ssDNAs. Under such scenario, the overall renaturation rate would inversely scale with the length of c-ssDNAs in a square root manner that is inconsistent with the experimental observations ${ }^{8}$. Here the sequence complexity is defined as the length of DNA with unique nucleotide sequence pattern (Fig. 1C). Several theoretical and computational models ${ }^{13,21-27}$ were developed recently to explain the observed scaling behaviours of the overall second order renaturation rate on the size of c-ssDNAs, temperature, ionic strength and viscosity of the reaction medium.

The nucleation step can be modelled as Kramer's escape problem over a free energy barrier ${ }^{23}$. In this model, it is argued that the square root dependency of nucleation rate on the length of c-ssDNA mainly originates from the entropic component of the free energy barrier. However, nature of the reaction coordinate and the entropic component of potential energy barrier associated with the renaturation process is unclear. According to the recently proposed three-step model ${ }^{15}$ (Scheme III in Fig. 2), the renaturation process comprises of (a) formation of nonspecific contact (b) nucleation or correct contact formation and (c) zipping. In the first step, c-ssDNAs perform three-dimensional collisions which result in the formation of Watson-Crick (WC) base pairs at random nonspecific contacts. Such nonspecific contacts randomly translocate along c-ssDNAs either via thermally driven one-dimensional (1D) slithering, inchworm movements and internal displacement ${ }^{24}$ mechanisms until finding the correct-contact to initiate the nucleation process. Nucleation will be followed by spontaneous zippering of c-ssDNAs. In this model, the square root dependency of the renaturation rate on the length of c-ssDNAs mainly originates from the non-specific contact formation step. However, it is still not clear whether the mode of nucleation is via 1D or 3D diffusion. It is also not clear how the inverse scaling on the sequence complexity arises in case of repetitive cssDNAs. Further, the retardation effects of the repetitive DNA sequences were not considered in Scheme III of Ref. ${ }^{15}$.

Understanding the role of the conformational state of DNA on the rate of hybridization is critical to unravel the underlying mechanism. To understand the equilibrium thermodynamic properties of the DNA hybridization, several course grained lattice models were developed and investigated using Monte Carlo simulation methods ${ }^{27}$. However, simulation of such systems with detailed base-paring and base-stacking interactions are limited to very short DNA sequences 24,27 . Such studies are useful to obtain the equilibrium thermodynamic properties rather than the nonequilibrium kinetic scaling aspects. Further, the kinetic scaling laws associated with the DNA hybridization are mainly dependent on the translational diffusion of c-ssDNAs along with their interpenetration dynamics rather than the fine details of base-pairing and base-stacking interactions. In this background, we model c-ssDNAs as selfavoiding random walks (SARW) confined in a cubic lattice box which represents the reaction 
Lattice model on DNA hybridization

volume under in vitro conditions. Using this lattice model, we will unravel the origin of various kinetic scaling relationships associated with the renaturation dynamics in detail.

\section{Theoretical Methods}

\subsection{Translational diffusion of c-ssDNAs}

We model c-ssDNAs (we denote them as ssDNA 1 and 2 as in Fig. 1A) as loosely packed and approximately spherical shaped nucleotide clusters with a radius of gyration $R_{g}$. The 3D collisions between these c-ssDNAs are catered via their translational diffusion. When the length of both these strands are equal to $L$, then one can approximately assume equal radius of gyration $R_{g}$ for both the strands. In this situation, the maximum possible steady state Smolochowski rate of 3D diffusion controlled collisions between these c-ssDNAs will be $k_{t}=$ $\frac{8 k_{B} T}{3 \eta} \phi^{28}$ (Fig. 1B). Here $k_{B}$ is the Boltzmann constant, $\eta$ is the viscosity coefficient of the medium, $T$ is the absolute temperature in degrees $K$ and $\phi \cong\left|\frac{\left(\kappa / 2 R_{g}\right)}{\exp \left(\kappa / 2 R_{g}\right)-1}\right| \exp \left(\frac{\kappa}{\lambda}\right){ }^{15,29}$ is the multiplication factor associated with the electrostatic repulsions between the negatively charged phosphate backbones of c-ssDNAs along with the shielding effects of solvent molecules and other ions present at the DNA-DNA interface under dilute conditions. Here $\kappa=$ $\frac{\xi_{1} \xi_{2} e^{2}}{\Omega k_{B} T}$ is the Onsager radius which is defined as the distance between the colliding c-SSDNA strands at which the overall electrostatic interaction energy will be equal to that of the background thermal energy $\left(1 k_{B} T\right), \xi_{1}, \xi_{2}$ are the overall charge numbers on the base clusters of strand 1 and $2, \lambda$ is the thickness of the ionic shell present over the charged groups of cssDNAs. Here $\kappa$ will be a positive quantity since there is a charge-charge repulsion between $c$-ssDNAs. Since the electrostatic interactions act only at short ranges, in general one finds that $|\kappa| \ll 2 R_{g}$ where the reaction radius is $2 R_{g}$. As a result, $\left|\frac{\left(\kappa / 2 R_{g}\right)}{\exp \left(\kappa / 2 R_{g}\right)-1}\right| \cong 1$ and therefore the multiplication factor $\phi \cong \exp \left(\frac{\kappa}{\lambda}\right)$ will be independent on the radius of gyration of cssDNAs. One also should note that $\kappa<0$ in case of site-specific DNA-protein interactions since the DNA binding domains of the DNA binding proteins are usually rich in positively charged amino acids. Under such conditions, $\phi$ will be dependent on $R_{g}{ }^{30}$.

In the calculation of $k_{t}$, we have assumed an absorbing boundary condition for the associated diffusion equation at the reaction radius that is approximately equals to $2 R_{g}$. By definition, this is the rate of arrival of the base clusters of c-ssDNAs within the reaction radius. Here $k_{t}$ does not enumerate the number of contacts formed upon such collisions and subsequent interpenetrations. We will show in the later sections that there is always a nonzero probability for the occurrence of zero-contacts upon collision and interpenetration of c-ssDNAs. Clearly, the translational diffusion brings c-ssDNAs inside the reaction volume and there is no physical confinement of the polymer within a closed space here. The reacting c-ssDNAs enter and exit the reaction volume freely by diffusion. We will show in the later sections that this assumption is essential since the radius of gyration of c-ssDNAs will be strongly dependent on the confinement volume.

\subsection{Interpenetration dynamics of c-ssDNAs}

With this background, the nucleation rate $\left(k_{N}\right)$ will be directly proportional to the number of correct contacts formed between the c-ssDNAs per second. On the other hand, the average number of correct contacts formed per second $\left(k_{c c}\right)$ will be equal to the number of collisions 
between the base clusters of c-ssDNAs per second $\left(k_{t}\right.$, measured in $\left.\mathrm{M}^{-1} \mathrm{~s}^{-1}\right)$ multiplied by the average number of correct contacts $\left(\left\langle n_{c c}\right\rangle\right)$ formed after each collision and interpenetration inside the reaction volume $(V)$ i.e. $k_{c c}=k_{t}\left\langle n_{c c}\right\rangle$. As result, one obtains $k_{N} \propto k_{t}\left\langle n_{c c}\right\rangle$. Only the correct-contact is the perfect registry match between the c-ssDNAs that can lead to nucleation and zipping. Apart from the correct contacts, several incorrect contacts (icc) can also be formed between the c-ssDNAs upon collision. When the c-ssDNAs are repetitive, then there are possibilities for the trap correct contacts (tcc) as described in Fig. 1C. Trap correct contacts possess registry matches within the repeats but they are all incorrect contacts in the view of the entire c-ssDNAs. Here tcc can lead to partial duplexes with single strand overhangs which are actually kinetic traps in the pathway of DNA renaturation. We denote the number of cc, icc and tcc as $n_{c c}, n_{i c c}, n_{t c c}$ respectively. Clearly, $n_{c c}, n_{i c c}, n_{t c c}$ are all random variables since they vary from collision to collision and interpenetrations. We denote the corresponding ensemble averages as $\left\langle n_{c c}\right\rangle,\left\langle n_{i c c}\right\rangle$ and $\left\langle n_{t c c}\right\rangle$ respectively. With these definitions, one can derive the following expressions.

$k_{c c} \cong k_{t}\left\langle n_{c c}\right\rangle ; k_{i c c} \cong k_{t}\left\langle n_{i c c}\right\rangle ; k_{t c c} \cong k_{t}\left\langle n_{t c c}\right\rangle$.

In these equations, $k_{c c}, k_{i c c}$ and $k_{t c c}$ are respectively the number of correct, incorrect and trapcorrect contacts formed between c-ssDNAs strands per second. Clearly, the nucleation rate $k_{N} \propto k_{c c}$. Various symbols and definitions used throughout this paper are summarized in Table 1. The correct-contacts can form anywhere on the entire stretch of c-ssDNAs with length equals to $L$ number of nucleotides (nt). Therefore, the probability of getting a correct contact upon each collision will be $p_{c c}=1 / L$. This also means that the average number of correct and incorrect contacts are approximately connected via $\left\langle n_{c c}\right\rangle \cong p_{c c}\left\langle n_{i c c}\right\rangle$. To understand various scaling behaviours of $\left\langle n_{c c}\right\rangle,\left\langle n_{i c c}\right\rangle$ and $\left\langle n_{t c c}\right\rangle$, we model c-ssDNAs as selfavoiding random walks (SARWs) confined in a lattice cube box that represents the reaction volume $V$ of the standard bimolecular collision model. In this setting, the volume of a monomeric unit is $v=1$ and volume occupied by the c-ssDNA strand of length $L$ will be $v L$. When the complexity of ssDNA is $c \mathrm{nt}$, then there will be at least $L / c$ number of repeating sequences in each c-ssDNA strand. Here we assume that the respective c-ssDNA base clusters have already arrived at the reaction volume through the 3D translational diffusion with rate $k_{t}$. The average number of $\mathrm{cc}$, icc and tcc can be obtained by repeated generation of two independent SARWs of length $L$ inside a fixed cubic lattice box with volume $V$. In each iteration, the number of $\mathrm{CC}$, icc and tcc will be counted and these counts are averaged over $10^{5}$ SARW trajectories. Detailed stochastic simulations with fixed $L$ for both the c-ssDNA strands and reaction volume $V$ as given in the Simulation Methods section revealed the following scaling relationships.

$\left\langle n_{c c}\right\rangle \cong \frac{v L}{V} ;\left\langle n_{i c c}\right\rangle \cong \frac{v L^{2}}{V} ;\left\langle n_{t c c}\right\rangle \cong \frac{v L^{2}}{c V}$

Here $v / \mathrm{V}$ is the probability of finding any nucleotide of c-ssDNAs inside $V$ upon each movement and $v L$ is the total chain volume. Upon assuming c-ssDNAs as a spherical shaped nucleotide clusters, the reaction space can be thought approximately as a sphere with radius of $2 R_{g}$ where $R_{g}$ is the average radius of gyration of the individual c-ssDNA. One can straightforwardly interpret these results as follows. Since $L$ number of monomers of the cssDNA of interest search for the complementary nucleotides on the other strand at the same 
time, one obtains $\left\langle n_{i c c}\right\rangle \cong \frac{v L^{2}}{V}$. Out of these $n_{i c c}$ numbers of incorrect contacts, the probability of finding the trap correct contacts among the repetitive c-ssDNAs will be 1 / c. Therefore, one obtains $\left\langle n_{t c c}\right\rangle=\left\langle n_{i c c}\right\rangle / c$. In the same way, the probability of finding the correct contact will be $1 / L$ that is independent on the number of repeats. From this one can deduce that $\left\langle n_{c c}\right\rangle=\left\langle n_{i c c}\right\rangle / L$. This expression for $\left\langle n_{t c c}\right\rangle$ will work only when $L$ is much higher than the sequence complexity $c$ so that $\left(\frac{L}{c}\right) \gg 1$. When $c \rightarrow L$, then there are always chances for the occurrence of partial repeats and one can obtain the approximation $\lim _{c \rightarrow L}\left\langle n_{t c c}\right\rangle \cong \frac{v\left(\bar{L}^{2}+l^{2}\right)}{c V}$ where $\bar{L}=c\left\lfloor\frac{L}{c}\right\rfloor$ is the length of c-ssDNAs containing full repeats and $l=L-c\left\lfloor\frac{L}{c}\right\rfloor$ is the length of c-ssDNAs with partial repeat. For example, when $L=20$ and $c=6$, then one obtains $\bar{L}=18$ and $I=2$. Here $\left\lfloor\frac{L}{c}\right\rfloor$ is the floor function operator. For example, $\left\lfloor\frac{20}{6}\right\rfloor=3$. When the main template strand length is fixed at $L$ and only the length of the probe $u$ is varied as in case of PCR reactions, then we find the following scaling relationships.

$\left\langle n_{c c}\right\rangle \cong \frac{v u}{V} ;\left\langle n_{i c c}\right\rangle \cong \frac{L v u}{V} ;\left\langle n_{t c c}\right\rangle \cong \frac{L v u}{c V}$

When the length of both c-ssDNAs are randomized within $(0, L)$ with equal probabilities $(=1$ $/ L$ ) resembling the uniformly sheared DNA ${ }^{8}$ so that the average length will be $\langle L\rangle=L / 2$, then one finds the following scaling relationships.

$\left\langle n_{c c}\right\rangle \cong \frac{v\langle L\rangle}{2 V}=\frac{v L}{4 V} ;\left\langle n_{i c c}\right\rangle \cong \frac{v\langle L\rangle^{2}}{V}=\frac{v L^{2}}{4 V} ;\left\langle n_{t c c}\right\rangle \cong \frac{v\langle L\rangle^{2}}{c V}=\frac{v L^{2}}{4 c V}$

When the complexity $c=L$, then irrespective of the sheared nature of c-ssDNAs the probability of finding the correct contact will be $p_{c c}=1 / L$. As a result, one obtains $\left\langle n_{c c}\right\rangle \cong p_{c c}\left\langle n_{i c c}\right\rangle=$ $L v / 4 V$ for the case of sheared DNA. When $c \rightarrow\langle L\rangle$ then one can obtain the approximation $\lim _{c \rightarrow\langle L\rangle}\left\langle n_{t c c}\right\rangle \cong \frac{v\left(\langle\bar{L}\rangle^{2}+\langle l\rangle^{2}\right)}{c V}$ where $\langle\bar{L}\rangle=c\left\lfloor\frac{\langle L\rangle}{c}\right\rfloor$ and $\langle l\rangle=\langle L\rangle-c\left\lfloor\frac{\langle L\rangle}{c}\right\rfloor$ by definition as in case of non-sheared and repetitive c-ssDNAs.

\subsection{Radius of gyration of c-ssDNAs}

The ensemble average of the radius of gyration of a chain molecule can be defined as $R_{g}=$ $\left\langle\left|\sqrt{\frac{\sum_{i, j=1}^{L}\left(\vec{w}_{i}-\vec{w}_{j}\right)^{2}}{2 L^{2}}}\right|\right\rangle$ where $\vec{w}_{i}$ is the vector coordinates $(X, Y, Z)$ of the $\mathrm{i}^{\text {th }}$ monomer and $L$ is the length ${ }^{31,32}$. Here the averaging is done over several possible spatial configurations of the polymer. Detailed numerical simulations suggested that the scaling of $R_{g}$ with $L$ deviates significantly from the standard scaling $R_{g} \propto \sqrt{L}$ when the ratio $\left(\frac{v L}{V_{S}}\right) \rightarrow 1$ where $V_{s}$ is the volume in which the SARW is confined. In other words, the scaling $R_{g} \propto \sqrt{L}$ will be valid only in the limit $\left(\frac{v L}{V_{S}}\right) \rightarrow 0$. In this context, nonlinear least square fittings of the values of $R_{g}$ obtained over various lengths of SARWs confined in different volumes $V_{s}$ using MarquartLevenberg algorithm ${ }^{33}$ suggested the following functional form.

$R_{g} \cong \alpha \sqrt{L}(1+\beta L)^{-1} ; \beta=\left(\varepsilon\left(\frac{v}{V_{S}}\right)^{\delta}+\sigma\right) ; \lim _{V_{S} \rightarrow \infty} R_{g} \cong \alpha \sqrt{L} ; \alpha \cong \sqrt{l_{p}^{2} / 6}$ 
Lattice model on DNA hybridization

Here $\alpha$ is the pre-exponent and $I_{p}$ is the physical distance between the monomers. Clearly, in the limit as $V_{S} \rightarrow \infty$ that represents the dilute in vitro conditions, we recover the well-known scaling relationship for a linear chain polymers as $R_{g} \cong l_{p} \sqrt{L / 6}$. The excluded volume effects will be prominent when the intrinsic volume of the polymer $v L$ is close to the confinement volume $V_{s}{ }^{8}$. Under such conditions, the interpenetration of c-ssDNAs among each other will be very much limited. In our model, c-ssDNAs are not physically confined in space. Rather, they are allowed to enter or exit the reaction volume freely. Hence, we assume $R_{g} \cong l_{p} \sqrt{L / 6}$ and subsequently derive the following expressions for the reaction and monomer volumes.

$V \cong \frac{4}{3} \pi\left(2 R_{g}\right)^{3}=\frac{32}{3} \pi l_{p}^{3}\left(\frac{L}{6}\right)^{3 / 2} ; v \cong \frac{4}{3} \pi l_{p}^{3} n_{D}^{3}$

In these equations, $I_{p} n_{D}$ is the radius of gyration of the nucleotide monomer in terms of number $\left(n_{D}\right)$ of distances between adjacent nucleotides $\left(l_{p} \cong 3.4 \times 10^{-10} \mathrm{~m}\right.$ for dsDNA and $l_{p} \cong(5.9$ to 7$) \times 10^{-10} \mathrm{~m}$ for c-ssDNAs, since $l_{p}$ corresponding to c-ssDNAs is a fluctuating quantity, we denote $l_{p}$ corresponding to dsDNA as the standard base-pair unit, bp) and the volumes are measured in $\mathrm{bp}^{3}$. When we model DNA as chain of beads where each bead represents a monomer, then the radius of the monomer bead will be approximately equal to the radius of the DNA cylinder which is approximately equals to $n_{D} l_{p} \approx 3 \mathrm{bp}$. Using Eqs. 6, when $L \gg c$, then one can derive the following expressions for the number of $\mathrm{cc}$, icc and tcc formed upon interpenetration of c-ssDNAs inside the reaction volume.

$\left\langle n_{c c}\right\rangle \cong \frac{3 \sqrt{6} n_{D}^{3}}{4 \sqrt{L}} ;\left\langle n_{i c c}\right\rangle \cong \frac{3 \sqrt{6} n_{D}^{3} \sqrt{L}}{4} ;\left\langle n_{t c c}\right\rangle \cong \frac{3 \sqrt{6} n_{D}^{3} \sqrt{L}}{4 c}$

When c-ssDNAs are equal in length, then using Eqs. $\mathbf{6}$ and $\mathbf{7}$ one can drive expressions for the overall rate associated with the formation of $\mathrm{cc}$, icc and tcc as follows.

$k_{c c} \cong k_{t} \frac{3 \sqrt{6} n_{D}^{3}}{4 \sqrt{L}} \propto \frac{1}{\eta \sqrt{L}} ; k_{i c c} \cong k_{t} \frac{3 \sqrt{6} n_{D}^{3} \sqrt{L}}{4} \propto \frac{\sqrt{L}}{\eta} ; k_{t c c} \cong k_{t} \frac{3 \sqrt{6} n_{D}^{3} \sqrt{L}}{4 c} \propto \frac{\sqrt{L}}{\eta c}$.

\section{4. One step DNA hybridization model}

Using the scaling results presented in Eqs. 7 and 8, we now revisit the Wetmur-Davidson model ${ }^{8}$. When the nucleation occurs via pure 3D diffusion controlled collision route as described in Scheme I of Fig. 1A, then the nucleation occurs with a rate $k_{N} \propto k_{c c}$ and one can straightforwardly derive the scaling as $k_{N} \propto \frac{1}{\eta \sqrt{L}}$. This means that, $k_{N}=\zeta k_{c c}$ where $\zeta$ is the dimensionless proportionality constant. In this background, the differential rate equations for the renaturation of a nonrepetitive c-ssDNAs can be written as follows.

$\frac{d\left[2 L M_{D}\right]}{d t}=k_{N}\left[L M_{1}\right]\left[L M_{2}\right] ; \frac{d\left[M_{D}\right]}{d t}=\left(\frac{k_{N} L}{2}\right)\left[M_{1}\right]\left[M_{2}\right] ; k_{R I} \cong\left(k_{c c} \zeta \frac{L}{2}\right) \cong k_{t} \zeta \frac{3 \sqrt{6} n_{D}^{3}}{8} \sqrt{L}$.

In this equation, we have substituted $k_{N}=\zeta k_{c c}$. Here $k_{R l}$ is the overall hybridization rate corresponding to Scheme I, $M_{D}$ (mol/lit) is the concentration of dsDNA molecules and $M_{1}$ and $M_{2}$ (mol/lit) are the concentrations of the c-ssDNA molecules. Upon multiplying by $L$ (or $2 \mathrm{~L}$ in case of dsDNA) one can convert these concentrations of the polymer molecules into the 
concentrations of nucleotides in each category which are actually the experimentally observed variables. In the derivation of Eqs. 9, one assumes that the zipping is spontaneous and the nucleation is the rate limiting step. Remarkably, Eqs. 9 associated with the WetmurDavidson model correctly predicts the length dependent scaling as $k_{R I} \propto \frac{\sqrt{L}}{\eta}$ in line with the experimental observations ${ }^{8,14}$.

\section{5. Two step mechanism of DNA hybridization}

When the concentration of the nucleus is $W$ and the average size of a nucleus is $N$ nt (so that the concentration of $\mathrm{nt}$ in the nucleus form dsDNA is [2NW]), then from the two-step mechanism given in Scheme II of Fig. 1A, one can derive the following rate equations.

$$
\begin{aligned}
& \frac{d[2 N W]}{d t}=k_{N}\left[L M_{1}\right]\left[L M_{2}\right]-k_{Z}[2 N W] ; \frac{d\left[2 L M_{D}\right]}{d t}=k_{Z}[2 N W] ; \frac{d W}{d t} \rightarrow 0 ; \frac{d\left[M_{D}\right]}{d t} \cong \\
& \left(\frac{k_{N} L}{2}\right)\left[M_{1}\right]\left[M_{2}\right] ; k_{R I I} \cong\left(\zeta k_{c c} \frac{L}{2}\right)
\end{aligned}
$$

In this equation, $k_{R I I}$ is the overall hybridization rate corresponding to Scheme II, $k_{z}(n t / s)$ is the zipping rate constant. When zipping is much faster than the nucleation step, then the rate equations given in Eqs. 10 attain steady state so that one can set $\frac{d W}{d t} \rightarrow 0$. Here nucleation will be the rate limiting step. In such scenario, Eqs. 10 reduce to Eqns. 9 where $k_{c c}=k_{i c c} / L$ for nonrepetitive c-ssDNA. Therefore one obtains the expression $k_{R I I} \cong\left(k_{c c} \zeta \frac{L}{2}\right) \propto \sqrt{L}$.

\section{6. Limitations of one step and two-step hybridization models}

The main flaws of Eqs. $\mathbf{9}$ and $\mathbf{1 0}$ are as follows.

1. Clearly, Eqns. $\mathbf{9}$ and $\mathbf{1 0}$ will work only for short DNA segments for which one can ignore the zipping times. However, for long c-ssDNAs, the zipping time $\tau_{z}$ scales with the length of DNA in a linear or square ${ }^{15}$ or power law manner ${ }^{26}$. In general, one observes the scaling for the zipping rate (which is the inverse of the zipping time) as $k_{Z} \propto L^{-\rho}$. There are two extreme possibilities. 1) When the zipping is diffusion like and not energetically driven or hampered by the chain entropy of the single stranded overhangs, then the zipping will be a diffusion like process and $k_{Z} \propto L^{-2}$. 2) When the zipping is irreversible and energetically driven process over the entropic barriers, then one finds that $k_{Z} \propto L^{-1}$. In reality, the chain entropy barrier decreases in the process of zipping and the stability of duplex increases along the transition from ssDNA to dsDNA form. In general, when the entropic barrier associated with the single strand overhangs is significant then one observes the exponent as $1 \leq \rho \leq 2$ depending on the type of prevailing conditions. For example, when the zipping is similar to that of the forced translocation of a polymer through a nanopore ${ }^{26}$, then one finds that $\rho \cong 1.37$.

2. When $L=1$, then one finds from the extrapolation of experimental data ${ }^{34}$ that $\lim _{L \rightarrow 1} k_{R I I} \cong 5 \times 10^{-3} k_{s m}$ where $k_{s m}=k_{t} / \phi$ is the maximum possible Smoluchowski diffusion controlled bimolecular collision rate limit across neutral molecules. However, Eqs. 9 and 10 associated with the 3D diffusion only model predict that $\lim _{L \rightarrow 1} k_{R I I} \cong$ $k_{s m} \phi \frac{3 \sqrt{6} n_{D}^{3}}{8}$. This means that the contributions from the electrostatic repulsions across 
Lattice model on DNA hybridization

the negatively charged phosphate backbones over the rate enhancement at the DNA-DNA interface of c-ssDNAs should be $\phi \cong \frac{4 \times 10^{-2}}{3 \sqrt{6} n_{D}^{3}}$ where $n_{D} \cong 3 \mathrm{nt}$.

3. When the mode of nucleation is via pure 3D diffusion, then the number of correctcontacts or the nucleation rate should be independent of the number of repeats in the cssDNA strands. Since the zipping step directly follows from the 3D diffusion mediated nucleation, the overall renaturation rate should be independent of the complexity of cssDNAs. Further, the presence of trap correct-contacts would slow down the nucleation process since tcc across the c-ssDNAs need to be broken before exploring other locations for the correct-contacts ${ }^{13,24}$. This incurs significant amount of time lapse which in turn delays the renaturation process. On the other hand, such tcc keeps the complementary strands in the close vicinity for an extended amount of time compared to icc. This is essential for the efficient operation of various one dimensional facilitating processes such as slithering, inchworm movements and internal displacement mechanisms ${ }^{13,24}$ which can speed up the rate of nucleation.

4. For repetitive ssDNA with complexity $c$ and $L / c$ number of repeats, Wetmur-Davidson model directly assumed the scaling $k_{R I I} \propto \frac{\sqrt{L}}{c \eta}$ which predicted that $k_{R I I} \propto \frac{1}{\sqrt{L}}$ at $c=L$ that is not consistent with their experimental observations ${ }^{8,14}$. This critical flaw and other arguments arising out of the simulation results ${ }^{24}$ clearly suggest that the nucleation process must involve at least two different pathways viz. 3D diffusion only mode and a mode with the combination of 1D and 3D diffusions ${ }^{15}$ as described in Schemes III and IV of Fig. 2. Here 3D diffusion only pathway progresses directly via correct-contacts and it works only for short c-ssDNAs where the zipping times can be ignored. The 3D-1D diffusion pathway can progress via either incorrect contacts or trap correct contacts depending on the repetitive nature of c-ssDNAs.

\section{7. Three step models of DNA hybridization}

According to Scheme III, the base clusters of c-ssDNAs interpenetrate each other upon collision to the form incorrect contacts in the first step with a rate $k_{i c c}$. Subsequently, the correct contact will be formed via various 1D facilitating processes such as slithering, inchworm movements and internal displacement mechanisms. These are all analogous to the facilitating processes such as sliding, hopping and intersegmental transfers exhibited by the DNA binding proteins in the process of searching for their cognate sites on DNA ${ }^{35}$. Nucleation occurs upon finding the correct-contacts over several rounds of incorrect-contacts, 1D slithering movements and dissociations. With this background, Eqs. 10 can be rewritten for the case of nonrepetitive c-ssDNAs as follows (Scheme III in Fig. 2).

$$
\begin{aligned}
& \frac{d[2 U Y]}{d t}=k_{i c c}\left[L M_{1}\right]\left[L M_{2}\right]-\left(k_{r}+k_{N Z}\right)[2 U Y] ; \frac{d\left[2 L M_{D}\right]}{d t}=k_{N Z}[2 U Y] ; \frac{d[Y]}{d t} \rightarrow 0 ; \frac{d\left[M_{D}\right]}{d t} \cong \\
& \frac{k_{N Z} k_{i c c} L}{2\left(k_{r}+k_{N Z}\right)}\left[M_{1}\right]\left[M_{2}\right] ; k_{R I I I} \cong \frac{k_{N Z} k_{i c c} L}{2\left(k_{r}+k_{N Z}\right)}=\frac{k_{i c c} L}{2\left(1+k_{r} \tau_{N Z}\right)}
\end{aligned}
$$

In these equations, $k_{R I I I}$ is the overall hybridization rate corresponding to Scheme III, [2UY] is concentration of the nucleotides involved in the icc, $k_{r}(1 / \mathrm{s})$ is the dissociation rate constant connected with the icc, $\tau_{N Z}=\frac{1}{k_{N Z}}$ is the total time required for the nucleation and zipping 
through incorrect-contact formation route and its inverse will be the overall nucleationzipping rate. After each incorrect-contact, c-ssDNAs perform 1D slithering on each other over $q$ nucleotides on an average and then dissociate. The time required for such 1D diffusion will be $\tau_{q} \cong \frac{l_{p}^{2} q^{2}}{6 D_{o}}{ }^{15}$. In this time, c-ssDNAs scan $q$ nt on each other for the presence of correct contacts. To completely scan the entire c-ssDNAs of length $L$, at least $L$ / $q$ numbers of such cycles of incorrect-contact formation, 1D slithering and dissociations are required. Therefore, the nucleation time can be expressed as $\tau_{N} \cong \frac{L \tau_{q}}{q}$ which is the minimum amount of time that is required by the c-ssDNAs to find a correct-contact. When c-ssDNAs are nonrepetitive, then one finds the total time required for the nucleation and zipping steps as $\tau_{N Z} \cong \tau_{Z}+\tau_{N}$ where $\tau_{Z} \cong \frac{L}{k_{+}}$and $\tau_{N} \cong \frac{l_{p}^{2} q L}{6 D_{o}}$ are the zipping and nucleation times through the incorrect contact route ${ }^{15}$. The nucleation rate will be the inverse $k_{N}=\frac{1}{\tau_{N}}$ and the zipping rate will be $k_{Z}=\frac{1}{\tau_{Z}}$. Noting that fact that nucleation will be immediately followed by zipping, we combine the nucleation and zipping times together.

Since the stabilizing effects of the already formed Watson-Crick base pairs are much stronger than the entropic barriers arising out of the freely moving single stranded overhangs, one can assume the linear scaling for the zipping time with the length of c-ssDNAs as $\tau_{Z} \propto L$. Here $k_{+}\left(\mathrm{nt} / \mathrm{s}\right.$ ) is the microscopic zipping rate (Fig. 2), $I_{p}=1 \mathrm{bp}, q(\mathrm{nt})$ is the average slithering length and $D_{O}\left(\mathrm{bp}^{2} / \mathrm{s}\right)$ is the phenomenological $1 \mathrm{D}$ diffusion coefficient associated with the slithering dynamics of c-ssDNA segments. Upon inserting the expressions for the nucleation and zipping times into the expressions for $k_{R I I I}$ as given in Eq. 11 one obtains the following result.

$k_{R I I I} \cong \frac{k_{i c c} L}{2\left(1+k_{r} \tau_{N Z)}\right)}=\frac{k_{t} \frac{3 \sqrt{6} n_{D}^{3} \sqrt{L}}{4} L}{2\left(1+k_{r}\left(\frac{L}{k_{+}}+\frac{l_{p}^{2} q L}{6 D_{o}}\right)\right)} \propto \frac{\sqrt{L}}{\eta}$

Here one should note that $D_{0}$ will be influenced only by the local viscosity at the DNA-DNA interface of the c-ssDNAs with icc or tcc and it will not be much influenced by the global viscosity that is connected with the translational diffusion of the entire c-ssDNA nucleotide cluster. In the presence of repetitive sequences, apart from icc and cc, trap correct contacts are also formed as shown in Scheme IV of Fig. 2. Clearly, the nucleation can occur via both icc and tcc in case of repetitive c-ssDNAs. Particularly, these tcc can influence the renaturation in two possible ways viz. 1) they keep the complementary strands in close vicinity for prolonged amount of time that enhances the slithering times which in turn increase the efficiency of searching for the correct contacts. 2) these kinetic traps prolong the overall renaturation timescales. As a result of these, the dissociation rate connected with the partial duplexes decreases $\left(k_{r} \rightarrow k_{r t}\right)$ and the slithering length increases $\left(q \rightarrow q_{t}\right)$. The overall renaturation rate corresponding to the tcc route can be obtained by replacing $\left(k_{r} \rightarrow k_{r t}, q \rightarrow q_{t}, k_{i c c} \rightarrow\right.$ $\left.k_{t c c}\right)$ in Eq. 12. Upon combining the contributions of these parallel pathways through icc and tcc routes, one finally obtains the following expression for the overall renaturation rate for the repetitive c-ssDNAs. 
Lattice model on DNA hybridization

$$
k_{R I V} \cong \frac{k_{i c c} L}{2\left(1+k_{r} \tau_{N Z}\right)}+\frac{k_{t c c} L}{2\left(1+k_{r t} \tau_{N Z R}\right)} \cong \frac{3 k_{t} \sqrt{6} n_{D}^{3} \sqrt{L}}{4}\left\{\frac{L}{2\left(1+k_{r}\left(\frac{L}{k_{+}}+\frac{l_{p}^{2} q L}{6 D_{o}}\right)\right)}+\frac{L}{2 c\left(1+k_{r t}\left(\frac{L}{k_{+}}+\frac{l_{p}^{2} q_{t} L}{6 D_{o}}\right)\right)}\right\}
$$

Eq. 13 is the central result of this paper. In this equation, $k_{R I V}$ is the overall hybridization rate corresponding to Scheme IV, $\tau_{N Z R} \cong \tau_{Z}+\tau_{N R}$ is the nucleation-zipping time via trap correct contact route where $\tau_{N R} \cong \frac{l_{p}^{2} q_{t} L}{6 D_{o}}, k_{r t}$ is the dissociation rate connected with tcc and $q_{t}$ is the 1D slithering length associated with the repetitive c-ssDNAs with partial duplexes and single strand overhangs. The nucleation rate will be the inverse $k_{N R}=\frac{1}{\tau_{N R}}$. When $L>c$, then $k_{r}>k_{r t}$ since the partial duplexes are more stable than the incorrect contacts and $q<q_{t}$ since the cssDNAs stay close to each other for prolonged amount of time which allows extended amount of slithering. As a result these, we find that $\left(k_{r}\left(\frac{1}{k_{+}}+\frac{l_{p}^{2} q}{6 D_{o}}\right)\right) \ll\left(k_{r t}\left(\frac{1}{k_{+}}+\frac{l_{p}^{2} q_{t}}{6 D_{o}}\right)\right)$ from which one obtains the scaling as $k_{R I V} \propto \frac{\sqrt{L}}{c \eta}$. Remarkably, when $c=L$, Eqs. 13 predicts the correct scaling $k_{R I V} \propto \frac{\sqrt{L}}{\eta}$ in line with the experimental observations ${ }^{8,14}$.

Eq. 13 will be still valid when c-ssDNAs are unequal in length similar to that of the template and probe DNAs in PCR reactions. However, in this case the length of duplex formed upon hybridization will be equal to the length of the probe. Let us denote the lengths of template and probe as $L$ and $u$ respectively. When $L \gg u$, then the template strand diffuses slower than the probe strand and one can show that the bimolecular collision rate scales as $k_{t} \propto \sqrt{\frac{L}{u}}$. This follows from the fact that $k_{t} \cong \frac{2 k_{B} T}{3 \eta} \frac{\left(R_{u}+R_{L}\right)^{2}}{R_{u} R_{L}}$ where $R_{u} \propto \sqrt{u}$ and $R_{L} \propto \sqrt{L}$ are the radius of gyration of the probe and template strands respectively ${ }^{28}$. When $u \ll L$, then the reaction volume will be almost independent on the volume of the probe. This results in the scaling as $k_{c c} \propto \frac{\sqrt{u}}{L}$ for the rate of formation of the correct contacts. This means that the nucleation rate scales with the lengths of the probe and template c-ssDNAs as $k_{N} \propto \frac{\sqrt{u}}{L}$ since $k_{N} \propto k_{c c}$ which reduces to the observed scaling $k_{N} \propto \frac{1}{\sqrt{L}}$ as $c \rightarrow L$.

\section{Simulation Methods}

To understand various scaling relationships associated with the correct, incorrect and trap correct contacts in the process of renaturation, we modelled the c-ssDNAs as self-avoiding random walks confined inside a 3D lattice box that mimics the reaction volume. When the box is a cube with side $b$, then the box volume will be $V=b^{3}$ cubic units and $(X, Y, Z)=(0, b)$ are the reflecting walls for the generation of SARW 1 and 2 which mimic c-ssDNA strands 1 and 2 . The SARWs were generated starting from a random point by sequentially calling three random number $r_{1,2,3} \in(0,1)$. If $r_{1}<0.5$, then $X \rightarrow X+1$ else $X \rightarrow X-1$ and similar rules were set for $Y$ and $Z$ dimensions. The move will be allowed only when the next location is not selfintersecting the earlier locations which mimics the implementation of the excluded volume effects. SARW 1 and SARW 2 cannot self-intersect but they can cross-intersect each other that mimics the interpenetration and contact formation among the pair of c-ssDNAs. 
Lattice model on DNA hybridization

When the sequence is repetitive with complexity $c$, then in such a polymer of length $L>c$, the positions from 1 to $c, c+1$ to $2 c$ and $2 c+1$ to $3 c$ and so on are identical in sequence. Those contacts between c-ssDNAs without exact registry matches are the incorrect contacts. The positions from 1 to $c$ of SARW 1 can form duplex with position stretch $c+1$ to $2 c$ of SARW 2 and so on. These cross intersections can be classified as correct contact (cc), incorrect contact (icc) or trap correct-contact (tcc) depending the position of the registry match. In this setting, the volume of a SARW with length $L$ will be $v L$ where $v=1$ is the volume of the monomer unit. In a repetitive c-sSDNA with complexity $c$, when there is a contact between position 1 of SARW 1 with position $\mathrm{c}+1$ of SARW 2 , then it is defined as a trap correct contact. When the crossintersection points are exact registry matches, then they are the correct-contacts. For example, when there is a contact between position 2 of SARW 1 with position 2 of SARW 2, then it is a correct contact. All other types of contacts are incorrect-contacts by definition.

To understand the effects of sheared c-ssDNAs on the overall rate of renaturation, we randomized the lengths of SARWs. Length of the original c-SSDNA is $L$ and we denote the index of the monomers from 1 to L. Shearing of c-ssDNAs will generate fragments of this template strand with random lengths. To mimic this, two random integers $r_{1}, r_{2}$, were generated inside $(1, L)$ which are the random lengths of SARW 1 and SARW 2 . The starting point of SARW 1 was defined by calling a random integer $s_{1}$ inside $\left(1, L-r_{1}\right)$ and the end point will be $s_{1}+r_{1}$. Similarly the starting point of SARW 2 was defined by calling random integer $s_{2}$ inside $\left(1, L-r_{2}\right)$ and the end point will be $s_{2}+r_{2}$. These start and end locations of SARW 1 and 2 were used to compute the number of correct, incorrect and trap correct contacts.

Here we assume that the pair of c-ssDNAs have already reached the reaction volume $V$ via 3D translational diffusion with a bimolecular collision rate $k_{t}$. The quantities that we are interested to compute here are the average number of correct, incorrect and trap correct contacts formed between a pair of c-ssDNAs of length $L$ and complexity $c$ inside the reaction volume $V$. To achieve this, several pairs of SARWs were generated inside a closed cubic lattice box as in Fig. 3 and the number of $\mathrm{Cc}$, icc and tcc were enumerated and averaged over $10^{5}$ trajectories. The average number of $c c$, icc and tcc seem to be influenced by the length of cssDNA $(L)$, its sequence complexity $(c)$ and the reaction volume $(V)$. To investigate the effects of these variables, we iterated one factor over a range of values at a time by fixing other two factors at constant values. In the real situations, one can translate the results of the lattice model by setting the reaction volume as the volume of the sphere with radius equals to $2 R_{g}$ where $R_{g}$ is the average radius of gyration of c-ssDNAs. To understand the distribution of icc, cc and tcc, we constructed the histogram of samples drawn from large population of counts over $10^{4}$ numbers of SARW trajectories.

\section{Results and Discussion}

The c-ssDNA strands can be modelled as self-avoiding random walks with the average radii of gyration $R_{g}$. Renaturation of c-ssDNAs requires the formation of correct contacts between them that leads to nucleation and zipping. Here c-ssDNAs reach the reaction volume via 3D translational diffusion. Upon entering the reaction volume, they can interpenetrate each other to form various types of contacts such as cc, icc, tcc or no contact at all. Clearly, when the size of c-ssDNAs are approximately equal then the rate at which they enter the reaction 


\section{Lattice model on DNA hybridization}

volume via translational diffusion $\left(k_{t}=\frac{8 k_{B} T}{3 \eta} \phi\right)$ will be independent of the radius of gyration. However, the extent of interpenetration of these strands will be strongly dependent on the length of c-ssDNAs $L$ and the confinement volume $V$.

Detailed lattice model simulations revealed the general scaling of various types of contacts between c-ssDNA strands as $n_{Q} \propto \frac{v L^{\gamma}}{V}$ for nonrepetitive c-ssDNAs where $\mathrm{Q}=$ (icc, cc) and $v$ is the monomer volume. For $\mathrm{cc}$, we find the exponent $\gamma=1$ and for icc we find that $\gamma=2$. For repetitive c-ssDNAs one finds that $n_{t c c} \propto \frac{v L^{\gamma}}{c V}$ with $\gamma=2$. When $c=L$, this reduces to $n_{c c} \propto \frac{v L^{\gamma-1}}{V}$ resembling the nonrepetitive case. Variations of the number of tcc, $c c$ and icc with respect to changes in $L$ with fixed $V$ and $c$ are demonstrated in Fig. 4. Variations of the number of tcc, cc and icc with respect to changes in $V$ with fixed $L$ and $c$ are demonstrated in Fig. 5. Results clearly suggest that the scaling $n_{Q} \propto \frac{v L^{\gamma}}{V}$ where $\mathrm{Q}=$ (icc, cc) is independent on the shape and dimension of the confining lattice box as shown in Figs. 4B-D and 5B-D. Similar results for the repetitive $c$-ssDNAs are demonstrated in Fig. 6A. We can conclude from these results that the number of correct contacts scales with $L$ and $V$ as $n_{c c} \propto \frac{v L}{V}$ irrespective of the complexity of c-ssDNA strands and shape and dimension of the confining lattice box.

These scaling relationships are not affected when the lengths of c-ssDNAs are unequal as demonstrated in Fig. 6B or random as demonstrated in Fig. 7. Particularly, when the length of the template SSDNA is $L$ and probe ssDNA is $u$ as in case of annealing phase of polymerase chain reactions, then the one obtains the scaling $n_{c c} \propto \frac{v u}{V}$. When the c-ssDNA lengths are random with equal distribution inside $(0, L)$, then one observes the scaling $n_{c c} \propto \frac{v\langle L\rangle}{2 V}$ where $\langle L\rangle=\frac{L}{2}$ here. These results are demonstrated in Fig. 7. Under in vitro conditions, $V$ will be the reaction volume. Since the reaction radius here is approximately equal to $2 R_{g}$, one can consider the reaction volume as $V \cong \frac{4}{3} \pi\left(2 R_{g}\right)^{3}$.

To understand the effect of varying the confinement volume on the radius of gyration, we iterated the length $L$ of SARWs at different confinement volumes. One naturally expects that the volume dependency of the scaling exponent $\theta$ in $R_{g} \propto L^{\theta}$ should be such that $\lim _{V_{S} \rightarrow \infty} \theta \rightarrow$ $\frac{1}{2}$. In this line, several asymptotic functions for the exponent were tried to fit the data on the computed $R_{g}$ versus the confinement volumes of SARWs. However, the volume and length dependency of $R_{g}$ seems to best fit the functional form $R_{g} \cong \alpha \sqrt{L}(1+\beta L)^{-1}$ where $\beta$ is a volume dependent parameter. Nonlinear least square fitting using Marquardt-Levenberg algorithm ${ }^{33}$ to this function revealed an approximate functional form for the parameter $\beta \cong$ $\left(\varepsilon\left(\frac{v}{V_{S}}\right)^{\delta}+\sigma\right)$ where $v$ is the monomer volume, $v_{S}$ is the confinement volume with the fit parameters $\varepsilon=0.5 \pm 0.04, \delta=0.61 \pm 0.1$ and $\sigma=-10^{-4} \pm 10^{-5}$ at $95 \%$ confidence level. These results are summarized in Figs. 8. The parameter $\alpha$ seems to be independent on $V_{\mathrm{S}}$ as shown in Fig. 8B. Clearly, one obtains the limiting condition $\lim _{V_{S} \rightarrow \infty} R_{g} \cong \alpha \sqrt{L}$ noting the fact that $\beta \rightarrow 0$ under such conditions as shown in Fig. $\mathbf{8 C}$. Since this limiting condition is valid under in vitro conditions, one can use this limiting expression to calculate the molecular and reaction volumes. Remarkably, the numbers of $\mathrm{cc}$, icc and tcc show bimodal type 
distribution with zero spike as demonstrated in Figs. 9. The reason for the zero-spike could be that the confinement volume here is much larger than the intrinsic volume of the c-ssDNA polymer. This mean that the magnitude of the zero-spike is inversely proportional to the volume ratio $v L / V$. The number of tcc decreases as the sequence complexity increases which is evident from Figs. 9C-F.

When the average radius of gyration of c-ssDNAs scales as $R_{g} \propto \sqrt{L}$, then the reaction volume scales with $L$ as $V \propto L^{3 / 2}$. As a result, one observes the scaling associated with the average number of correct contacts formed between the c-ssDNAs upon interpenetration as $\left\langle n_{c c}\right\rangle \propto$ $\frac{1}{\sqrt{L}}$. Since the rate of nucleation is directly proportional to the number of correct-contacts, one observes $k_{N} \propto \frac{1}{\sqrt{L}}$ in line with the experimental observations. When c-ssDNAs reach the reaction volume element via 3D translational diffusion, then one finally obtains the viscosity dependence as $k_{N} \propto \frac{1}{\eta \sqrt{L}}$. The overall renaturation rate in case of pure 3D diffusion model (Scheme I of Fig. 1A) is directly proportional to the nucleation rate as well as $L$. As a result, we finally arrive at the scaling for overall renaturation rate as $k_{R I} \propto \frac{\sqrt{L}}{\eta}$ in line with the experimental observations ${ }^{8}$ on the hybridization of nonrepetitive c-ssDNAs.

In case of repetitive c-ssDNAs, the average number of correct-contacts is independent on the sequence complexity or the number of repeating elements since it requires only the exact registry match. Clearly, models based on only the 3D diffusion cannot explain the complexity dependence of the renaturation rate. For example, when we assume the scaling $k_{R I} \propto \frac{\sqrt{L}}{c \eta}$ as in case of Wetmur-Davidson model, then we will end up with the inconsistency when $c \rightarrow L$ which predicts the scaling $k_{R I} \propto \frac{1}{\sqrt{L}}$ that is not in line with the experimental observations ${ }^{8}$. These arguments clearly suggest that the renaturation follows multiple and parallel pathways in the presence of repetitive sequences. Particularly, the 1D slithering dynamics plays critical roles in the renaturation of the repetitive sequences. The trap correct contacts between the repetitive c-ssDNAs can lead to the formation of partial duplexes with single strand overhangs. Although the entropy component of the single strand overhangs will destabilize these kinetic traps in the pathway of renaturation, tcc keep the c-ssDNAs in the close vicinity for a prolonged timescale that is essential for efficient slithering dynamics and internal displacement mechanisms which in turn accelerates the search for the correct contacts. Clearly, the extent of possible slithering dynamics is directly proportional to the number of trap correct contacts which is inversely proportional to the sequence complexity.

The pathway of renaturation via incorrect contact route always operates irrespective of the presence of repeating elements. Therefore, the overall renaturation rate associated with the repetitive c-ssDNAs will be the sum of rates corresponding to the incorrect contact and trap correct contact routes as described in Eq. 13. When the sequence complexity is close to the length of the c-ssDNAs, then the number of tcc and the associated slithering lengths will be much limited. This follows from the fact that the stability of incorrect contacts will be much lower than the trap correct contacts. As a result, the pathway via incorrect contacts will be the dominating one when $c$ is close to $L$ that is characterized by the scaling $k_{R I V} \propto \frac{\sqrt{L}}{\eta}$. The number of tcc and the extent of slithering lengths will be much high when the sequence 
complexity is much lesser than the length of c-ssDNAs. Under such conditions, the pathway via trap correct contacts will be the dominating one which is characterized by the scaling $k_{R I V} \propto \frac{\sqrt{L}}{c \eta}$ in line with the experimental observations ${ }^{8}$.

\subsection{Assumptions and limitations}

Although the presented lattice model could recover several kinetic scaling laws associated with the DNA renaturation dynamics, there are several assumptions and limitations viz. 1) the rigidities of both c-ssDNAs and dsDNA are ignored. 2) same intermonomer distance for both c-ssDNA and dsDNA strands were assumed. 3) detailed base-paring and base stacking interactions were ignored. 4) formation of intra strand loops were ignored and (5) GC base compositions of the c-ssDNA were not considered.

Translation diffusion of c-ssDNAs and their interpenetration upon collision mainly decide the kinetic scaling laws rather than the fine details of base-pairing and base-stacking interactions. Therefore, these factors will not affect the main scaling results much. Variation in the GC composition of c-ssDNAs will influence the microscopic zipping rates $k_{+}$and $k_{-}$-and subsequently the overall zipping rate $k$. However, this will not affect the scaling of the zipping rate with the length of c-ssDNAs. Variation of the intermonomer distances of c-ssDNAs will not affect the obtained scaling laws since we measure the lengths in terms of number of monomer units (nt). For example, we consider only the number of nucleotides scanned by the c-ssDNAs over slithering events rather than the number of intermolecular distances. The rigidity of both c-ssDNA and dsDNA will be mainly compensated by the conformational fluctuations driven by the chain entropy ${ }^{36}$. Formation of intra strand loop structures will influence our results in two different ways viz. 1) significant fraction of c-ssDNA segments will not be exposed for the exploration of correct contacts which in turn increases the time required for nucleation. 2) upon forming correct contacts at the non-loop regions, zipping will be hindered by the presence of intra strand loops which in turn increases the overall zipping time. This means that additional time components will be added up to the overall nucleation an zipping times that will not change the overall kinetic scaling laws much.

\section{Conclusion}

We have developed a lattice model on the rate of hybridization of the complementary sSDNA (c-ssDNA) strands. These c-ssDNAs can be thought as loosely packed and spherical shaped nucleotide clusters and the collisions between these clusters are catered via the threedimensional translational diffusion. Upon each collision, the base clusters of c-ssDNAs interpenetrate each other to form three different types of contacts among them viz. correct, incorrect and trap-correct contacts. Correct contacts are those with exact registry matches which can lead to nucleation and zipping of c-ssDNAs. Incorrect contacts are the mismatch contacts which are less stable compared to the trap correct contacts which can occur in the repetitive c-ssDNAs. Trap correct contacts possess exact registry match within the repeats. However, they are incorrect contacts in the view of the whole c-ssDNAs.

Trap correct contacts can form partial duplexes with single stranded overhangs. The nucleation rate $\left(k_{N}\right)$ is directly proportional to the average number of correct-contacts $\left(\left\langle n_{c c}\right\rangle\right)$ formed when c-ssDNAs interpenetrate each other inside the reaction volume. Here the cssDNAs reach the reaction volume via translational diffusion with rate $k_{t}$. This rate will be 
independent on the length of c-ssDNAs when both the c-ssDNAs are equal in size. As a result, the nucleation rate will be directly proportional to $k_{t}$ times the number of correct contacts. For short c-ssDNAs, the zipping times will much lower than the nucleation times. In such conditions, the overall renaturation rate $\left(k_{R}\right)$ will be directly proportional to $k_{N}$ and $L$ as $k_{R}=$ $k_{N} L \propto k_{t}<n_{c c}>L$ which is the 3D diffusion model.

To understand the scaling properties of the average number of various types of contacts with respect to the length $(L)$ of c-ssDNAs, sequence complexity $(c)$, and reaction volume $(V)$, we modelled the c-ssDNAs as a pair of self-avoiding random walks confined in a cubic lattice box which resembles the reaction volume $(V)$. Our lattice model simulations suggested the scaling $\left\langle n_{c c}\right\rangle \cong \frac{v L}{V},\left\langle n_{t c c}\right\rangle \cong \frac{v L^{2}}{c V}$ and $\left\langle n_{i c c}\right\rangle \cong \frac{v L^{2}}{V}$ where the $v=1$ is the monomer volume, $\left\langle n_{Q}\right\rangle$ are the average number of correct $(Q=c c)$, incorrect $(Q=i c c)$ and trap correct contacts $(Q=t c c)$. Further numerical analysis and nonlinear least square fitting results suggested the scaling for the average radius of gyration of c-ssDNAs with their length as $R_{g} \propto \sqrt{L}$. Since the reaction space will be approximately a sphere with radius equals to $2 R_{g}$, one obtains the scaling for the nucleation rate with length of c-ssDNA as $k_{N} \propto \frac{1}{\sqrt{L}}$ and one finally obtains $k_{R} \propto \sqrt{L}$ in line with the experimental observations. However, this expression works only for a nonrepetitive and short c-ssDNAs. When c-ssDNAs are repetitive with a complexity of $c<L$, then earlier models suggested the scaling $k_{R} \propto \frac{\sqrt{L}}{c}$. This scaling will break down when $c=L$. These observations clearly suggested the existence of at least two different pathways of renaturation viz. through incorrect contact and trap correct contact.

The trap correct contacts occurring between repetitive c-ssDNAs can lead to the formation of partial duplexes with single strand overhangs. These partial duplexes keep the c-ssDNAs in close vicinity for prolonged timescales which is essential for the extended 1D slithering that can speed up the searching for the correct contacts. Clearly, the extent of slithering dynamics will be inversely proportional to the sequence complexity $c$. When the complexity is close to the length of c-ssDNAs, then the pathway through incorrect contact will be the dominating one with minimal level of slithering. When the complexity is much lesser than the length of cssDNAs, then the pathway via the trap correct contact will be the dominating one.

\section{Conflict of Interest statement}

The author declares no conflict of interest. 
bioRxiv preprint doi: https://doi.org/10.1101/2021.12.22.473940; this version posted December 23, 2021. The copyright holder for this preprint (which was not certified by peer review) is the author/funder, who has granted bioRxiv a license to display the preprint in perpetuity. It is made available under aCC-BY-ND 4.0 International license.

Lattice model on DNA hybridization

\section{References}

1. Alberts, B., Molecular biology of the cell. Garland Science: New York, 2002.

2. Lewin, B.; Krebs, J. E.; Kilpatrick, S. T.; Goldstein, E. S.; Lewin, B., Lewin's genes X. Jones and Bartlett: Sudbury, Mass., 2011.

3. Duggan, E. L., Deformation of DNA. III. The effect of glycol and glycerol on the ultraviolet absorbances of DNA. Renaturation by dilution. Biochem Biophys Res Commun 1961, 6, 93-9.

4. Cavalieri, L. F.; Small, T.; Sarkar, N., The renaturation of denatured DNA. Biophys J 1962, 2, 339-50.

5. Maniatis, T.; Fritsch, E. F.; Sambrook, J., Molecular cloning : a laboratory manual. Cold Spring Harbor Laboratory: Cold Spring Harbor, N.Y., 1982; p x, 545 p.

6. Woo, S.; Rothemund, P. W., Self-assembly of two-dimensional DNA origami lattices using cation-controlled surface diffusion. Nat Commun 2014, 5, 4889.

7. Maune, H. T.; Han, S. P.; Barish, R. D.; Bockrath, M.; Goddard, W. A., III; Rothemund, P. W.; Winfree, E., Self-assembly of carbon nanotubes into two-dimensional geometries using DNA origami templates. Nat Nanotechnol 2010, 5 (1), 61-6.

8. Wetmur, J. G.; Davidson, N., Kinetics of renaturation of DNA. J Mol Biol 1968, 31 (3), 349-70.

9. Rau, D. C.; Klotz, L. C., A unified theory of nucleation-rate-limited DNA renaturation kinetics. Biophys Chem 1978, 8 (1), 41-51.

10. Chilton, M. D., Theoretical explanation of mouse satellite DNA renaturation kinetics. Nat New Biol 1973, 246 (149), 16-7.

11. Murugan, R., A stochastic model on DNA renaturation kinetics. Biophys Chem 2003, 104 (3), 535-41.

12. Sambriski, E. J.; Schwartz, D. C.; de Pablo, J. J., A mesoscale model of DNA and its renaturation. Biophys J 2009, 96 (5), 1675-90.

13. Sambriski, E. J.; Ortiz, V.; de Pablo, J. J., Sequence effects in the melting and renaturation of short DNA oligonucleotides: structure and mechanistic pathways. J Phys Condens Matter 2009, 21 (3), 034105.

14. Studier, F. W., Effects of the conformation of single-stranded DNA on renaturation and aggregation. $J$ Mol Biol 1969, 41 (2), 199-209.

15. Niranjani, G.; Murugan, R., Theory on the Mechanism of DNA Renaturation: Stochastic Nucleation and Zipping. PLoS One 2016, 11 (4), e0153172.

16. Galau, G. A.; Britten, R. J.; Davidson, E. H., Studies on nucleic acid reassociation kinetics: rate of hybridization of excess RNA with DNA, compared to the rate of DNA renaturation. Proc Natl Acad Sci U S A 1977, 74 (3), 1020-3.

17. Smith, M. J.; Britten, R. J.; Davidson, E. H., Studies on nucleic acid reassociation kinetics: reactivity of single-stranded tails in DNA-DNA renaturation. Proc Natl Acad Sci U S A 1975, 72 (12), 4805-9.

18. Subirana, J. A., Kinetics of renaturation of denatured DNA. II. Products of the reaction. Biopolymers 1966, 4 (2), 189-200.

19. Subirana, J. A.; Doty, P., Kinetics of Renaturation of Denatured DNA. I.

Spectrophotometric results. Biopolymers 1966, 4 (2), 171-87.

20. Thrower, K. J.; Peacocke, A. R., The kinetics of renaturation of DNA. Biochim Biophys Acta 1966, 119 (3), 652-4.

21. Araque, J. C.; Robert, M. A., Lattice model of oligonucleotide hybridization in solution. II. Specificity and cooperativity. J Chem Phys 2016, 144 (12), 125101. 
bioRxiv preprint doi: https://doi.org/10.1101/2021.12.22.473940; this version posted December 23, 2021. The copyright holder for this preprint (which was not certified by peer review) is the author/funder, who has granted bioRxiv a license to display the preprint in perpetuity. It is made available under aCC-BY-ND 4.0 International license.

Lattice model on DNA hybridization

22. Sanstead, P. J.; Tokmakoff, A., A lattice model for the interpretation of oligonucleotide hybridization experiments. J Chem Phys 2019, 150 (18), 185104.

23. Sikorav, J. L.; Orland, H.; Braslau, A., Mechanism of thermal renaturation and hybridization of nucleic acids: Kramers' process and universality in Watson-Crick base pairing. J Phys Chem B 2009, 113 (12), 3715-25.

24. Ouldridge, T. E.; Sulc, P.; Romano, F.; Doye, J. P.; Louis, A. A., DNA hybridization kinetics: zippering, internal displacement and sequence dependence. Nucleic Acids Res 2013, 41 (19), 8886-95.

25. Ferrantini, A.; Baiesi, M.; Carlon, E., A lattice polymer study of DNA renaturation dynamics. J Stat Mech-Theory E 2010.

26. Ferrantini, A.; Carlon, E., Anomalous zipping dynamics and forced polymer translocation. J Stat Mech-Theory E 2011.

27. Cumberworth, A.; Reinhardt, A.; Frenkel, D., Lattice models and Monte Carlo methods for simulating DNA origami self-assembly. J Chem Phys 2018, 149 (23), 234905.

28. Atkins, P. W., Physical chemistry. W.H. Freeman: San Francisco, 1978.

29. Debye, P., Reaction Rates in Ionic Solutions. Transactions of The Electrochemical Society 1942, 82 (1), 265.

30. Niranjani, G.; Murugan, R., Theory on the mechanism of site-specific DNA-protein interactions in the presence of traps. Phys Biol 2016, 13 (4), 046003.

31. Doi, M.; Edwards, S. F., The theory of polymer dynamics. Clarendon Press: Oxford [Oxfordshire], 1988.

32. Gennes, P.-G. d., Scaling concepts in polymer physics. Cornell University Press: Ithaca, N.Y., 1979.

33. Marquardt, D. W., An Algorithm for Least-Squares Estimation of Nonlinear Parameters. Journal of the Society for Industrial and Applied Mathematics 1963, 11 (2), 431441.

34. Wetmur, J. G., Excluded volume effects on the rate of renaturation of DNA. Biopolymers 1971, 10 (4), 601-13.

35. Niranjani, G.; Murugan, R., Generalized theory on the mechanism of site-specific DNA-protein interactions. J Stat Mech-Theory E 2016.

36. Murugan, R., Theory on the looping mediated directional-dependent propulsion of transcription factors along DNA. J Stat Mech-Theory E 2020, 2020 (1). 


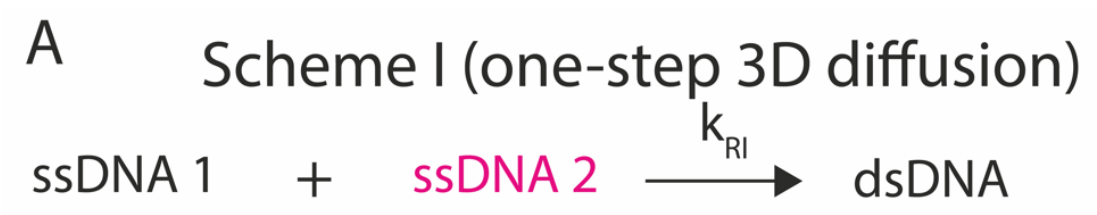

Scheme II (two-step 3D diffusion - zipping)

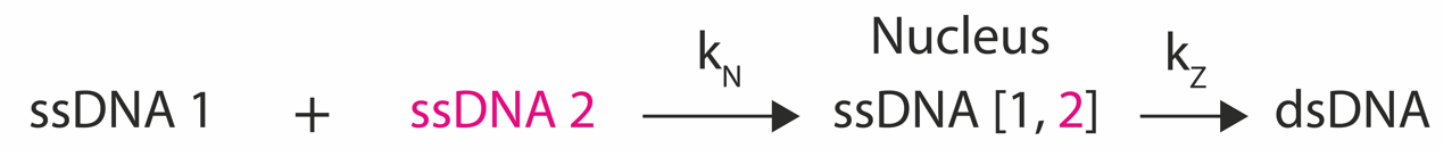

B

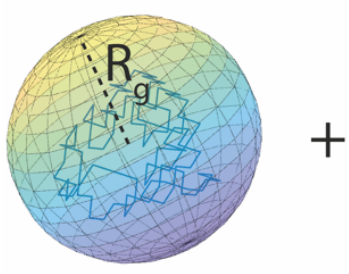

ssDNA 1

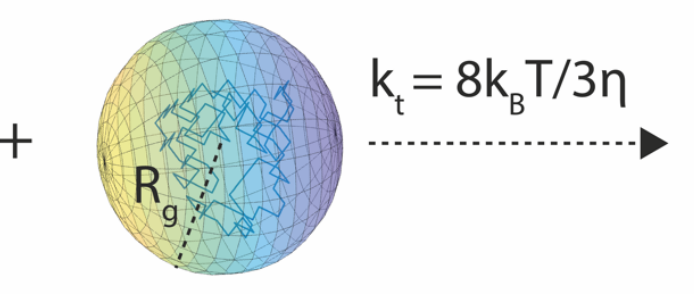

ssDNA 2

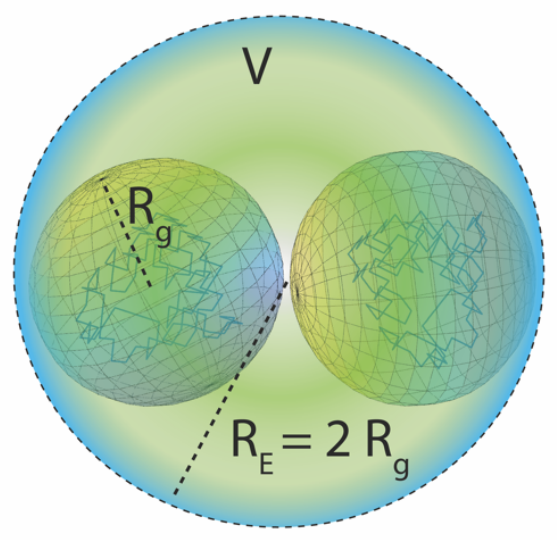

C

\section{5'-ATGAT ATGAT ATGAT ATGAT ATGAT ATGAT-3' 3'-TACTA TACTA TACTA TACTA TACTA TACTA-5' dsDNA}

\section{5'ATGAT ATGAT ATGAT ATGAT ATGAT ATGAT-3' ssDNA 1 \\ tcc \\ CC \\ 3'-TACTA TACTA TACTA 'TACTA TACTA TACTA-5' ssDNA 2}

FIGURE 1. Models on DNA renaturation. A. one-step, two-step models. In one-step model described in Scheme I, the complementary strands forms duplex with a second order rate $k_{R I}$ directly by diffusion controlled 3D collisions. In the two-step model described in Scheme II, the renaturation progress by the formation of stable nucleus with a rate $k_{N}$ via 3D diffusion mediated collisions and interpenetrations of c-ssDNAs and then subsequently by zipping with a rate $k_{z}$. B. The complementary ssDNA strands can be thought as spherical-shaped and loosely-packed nucleotide clusters with average radius of gyration $R_{g}$. Upon translational diffusion, they arrive inside the reaction volume $\mathrm{V}$ with a rate $k_{t}$ and interpenetrate each other. Here the reaction space is assumed to be a spherical one with radius $2 R_{g}$. When the complementary strands are confined inside $V$, there may be contacts between them. $C$. When 
bioRxiv preprint doi: https://doi.org/10.1101/2021.12.22.473940; this version posted December 23, 2021. The copyright holder for this preprint (which was not certified by peer review) is the author/funder, who has granted bioRxiv a license to display the preprint in perpetuity. It is made available under aCC-BY-ND 4.0 International license.

Lattice model on DNA hybridization

the contact occurs with exact registry match, then it is a correct-contact that can lead to nucleation and zipping. When contacts occur between nonidentical registers, then they are all incorrect contacts. When c-ssDNAs contain repeats, then there may be contacts between identical registries of repeats placed at two nonidentical locations. These are trap correctcontacts which can lead to the formation of partial duplexes with single strand overhangs. In the given example, there are six repeats. When the position 1 of first repeat interact with position 1 of second repeat, then it is a tcc. Sequence complexity is defined as the number of bases in an unique sequence. For example, the given sequence contains the repeats of 5 bases. Therefore, its complexity is $c=5$ where the total length is $L=30$. 


\section{Scheme III (three-step 3D-1D diffusion, nonrepetitive DNA)} ssDNA $1+$ ssDNA $2 \underset{\mathrm{k}_{\mathrm{r}}}{\stackrel{\mathrm{k}_{\mathrm{icc}}}{\rightleftarrows}}$ ssDNA $[1,2]_{\mathrm{icc}} \stackrel{\mathrm{k}_{\mathrm{N}}}{\longrightarrow}$ ssDNA [1, 2] $\stackrel{\mathrm{k}_{\mathrm{z}}}{\longrightarrow}$ dsDNA

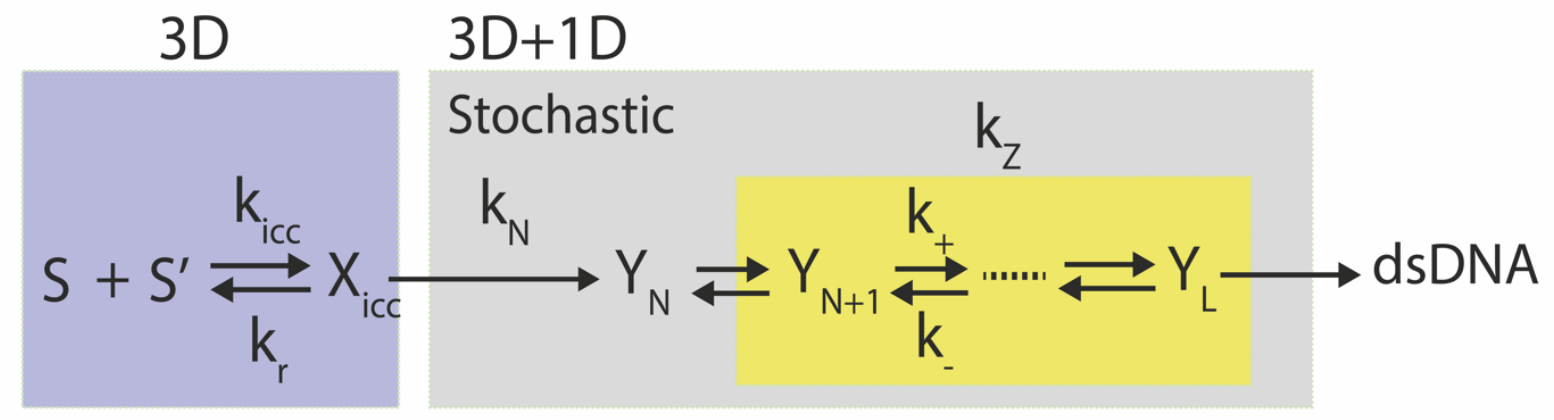

Scheme IV (three-step 3D-1D diffusion, repetitive DNA)
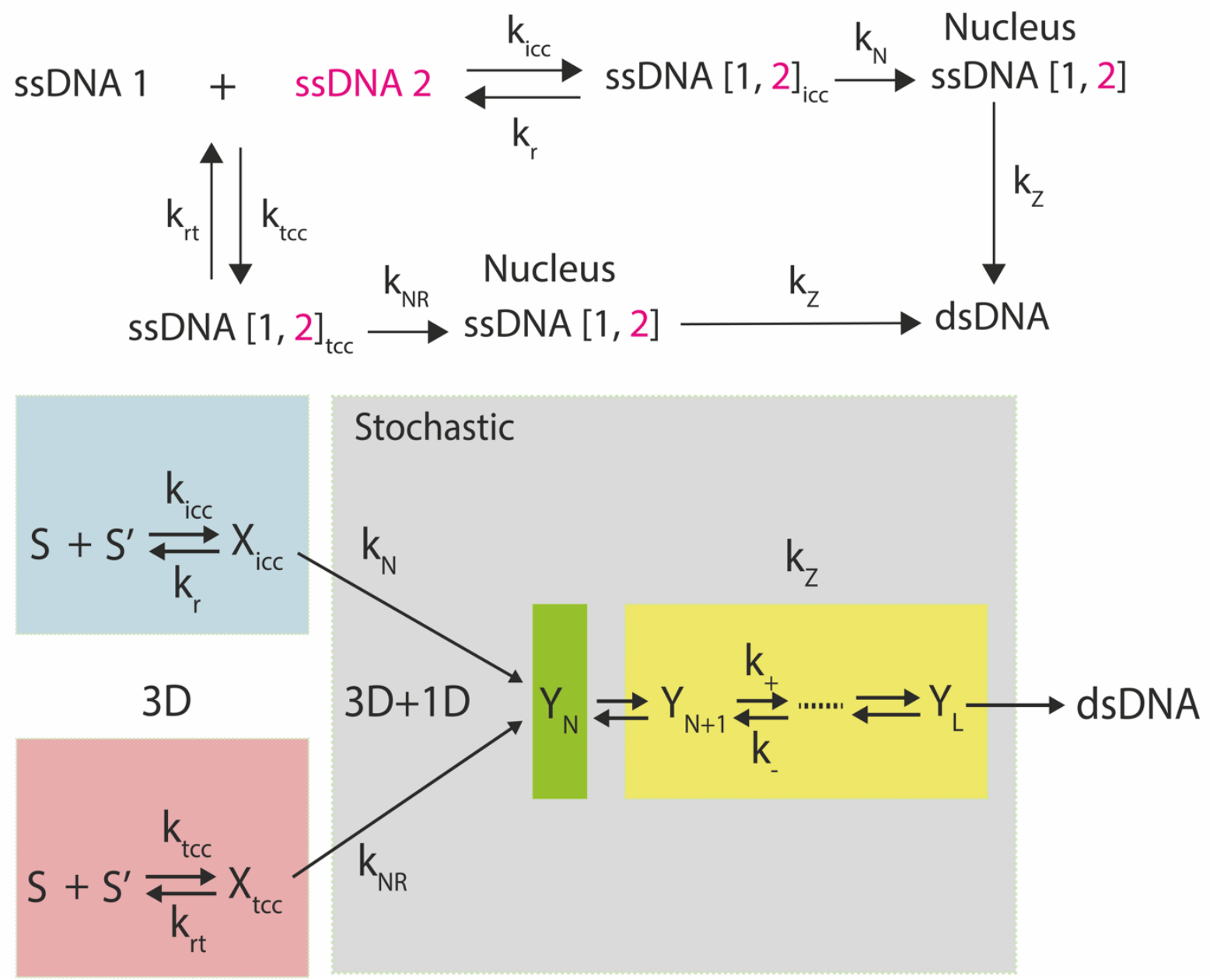

FIGURE 2. Three step models on DNA renaturation. Here $S$ and $S^{\prime}$ represents SSDNA1 and ssDNA2 respectively, $X_{i c c}=$ ssDNA $[1,2]_{i c c}, X_{t c c}=\operatorname{ssDNA}[1,2]_{\mathrm{tcc}}, Y_{N}$ represents the nucleus with $N$ nucleotides. In the three-step model given by Scheme III, c-ssDNAs renature via a combination of 3D and 1D diffusions. Here $k_{t c c}$ and $k_{i c c}$ are the number of incorrect and trapcorrect contacts formed per second, $k_{r}$ and $k_{r t}$ are respective dissociation rate constants, $k_{N}$ 
bioRxiv preprint doi: https://doi.org/10.1101/2021.12.22.473940; this version posted December 23, 2021. The copyright holder for this preprint (which was not certified by peer review) is the author/funder, who has granted bioRxiv a license to display the preprint in perpetuity. It is made available under aCC-BY-ND 4.0 International license.

Lattice model on DNA hybridization

and $k_{N R}$ are the respective nucleation rates and $k_{+}$an $k_{-}$are the forward and reverse microscopic rate constants associated with the zipping steps. In Scheme III, 3D collisions result in the formation of incorrect contacts which bring the strands into the close vicinity. Nucleation occurs via various 1D diffusion dynamics such as slithering, inchworm movements and internal displacement mechanisms. When c-ssDNAs are repetitive, then there is always chances for the formation of partial duplexes with single strand overhangs. This will in turn retain the c-ssDNAs in the close vicinity for prolonged amount of time that results in $k_{r}>k_{r t}$ and long slithering lengths. This will be a parallel pathway along with Scheme III as demonstrated in Scheme IV in case of repetitive c-ssDNAs. 


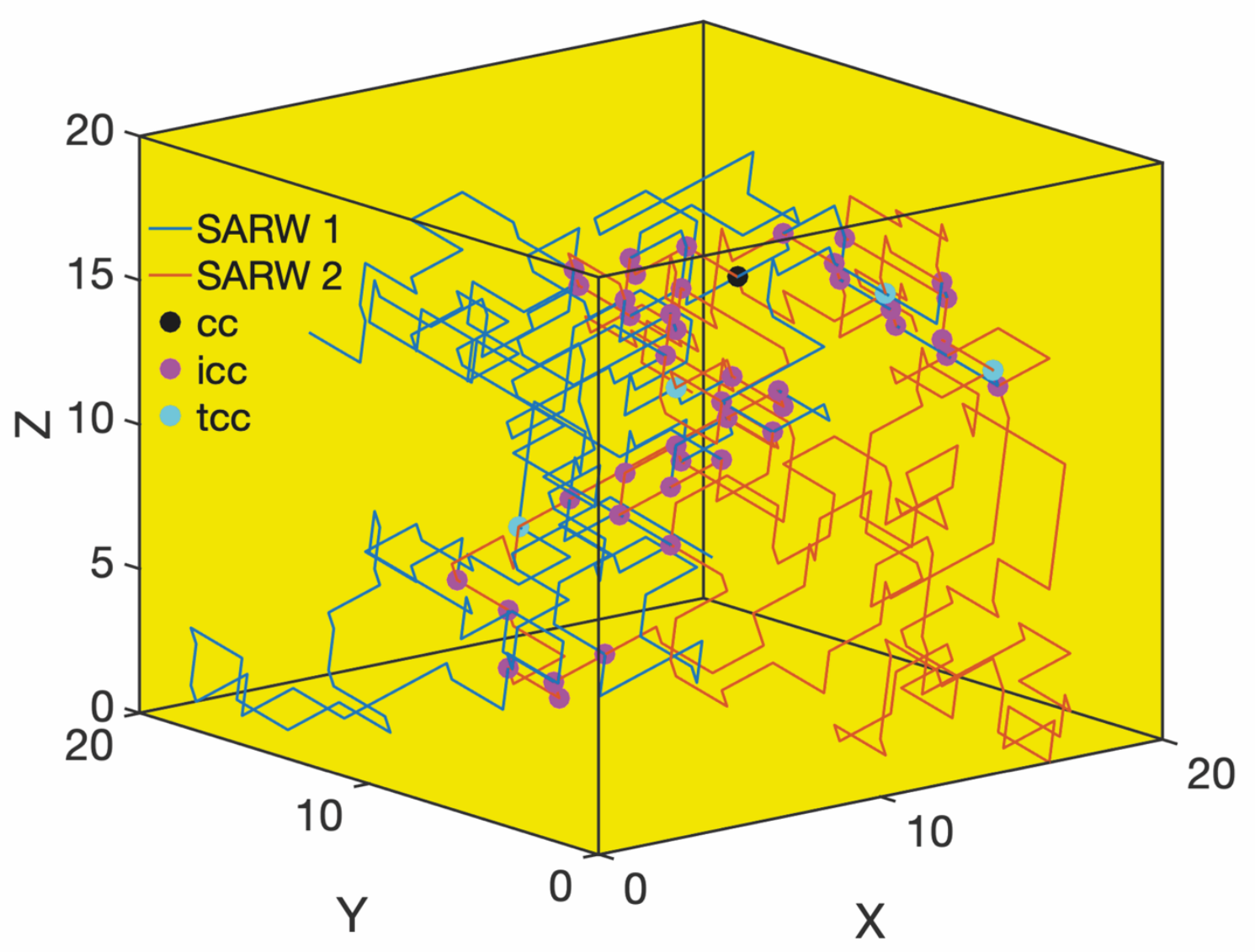

FIGURE 3. Lattice model on DNA renaturation. Here the complementary single strands are modelled as self-avoiding random walks (SARW 1 (blue) and 2 (red)) confined inside the reaction volume defined by the lattice box. The position of the nucleotides are marked from 1 to $L$. Both the strands arrive at the reaction volume via 3D diffusion. Correct contact between these SARWs occurs when there is an exact registry match that can further leads to nucleation and zipping. For example, when there is a contact between position 7 of SARW 1 with position 7 of SARW 2, then it is defined as a correct-contact (cc). When there is a contact between position 7 of SARW 1 with position 5 of SARW, then it is an incorrect contact (icc). When the complexity is $c<L$, then the SARW has $L / c$ number of repeats i.e. the sequence spanned across position 1 to $c, c+1$ to $2 c$ and $2 c+1$ to $3 c$ and so on will be the same. Therefore, when there is a contact between position 1 of SARW 1 with position $c+1$ (or $2 c+$ 1 and so on) of SARW 2, then it is defined as a trap correct-contact (tcc). Here tcc can lead to the formation of partial duplexes with single stranded overhangs which are actually kinetic traps in the renaturation pathway. Here the settings are, $L=250, c=5$, and reaction volume $V=20^{3}$, number of incorrect contacts $\left(n_{i c c}\right)=25$, trap correct contacts $n_{t c c}=4$ and the number of correct contacts $n_{c c}=1$ that occurs at $(X, Y, Z)=(9,8,17)$. 

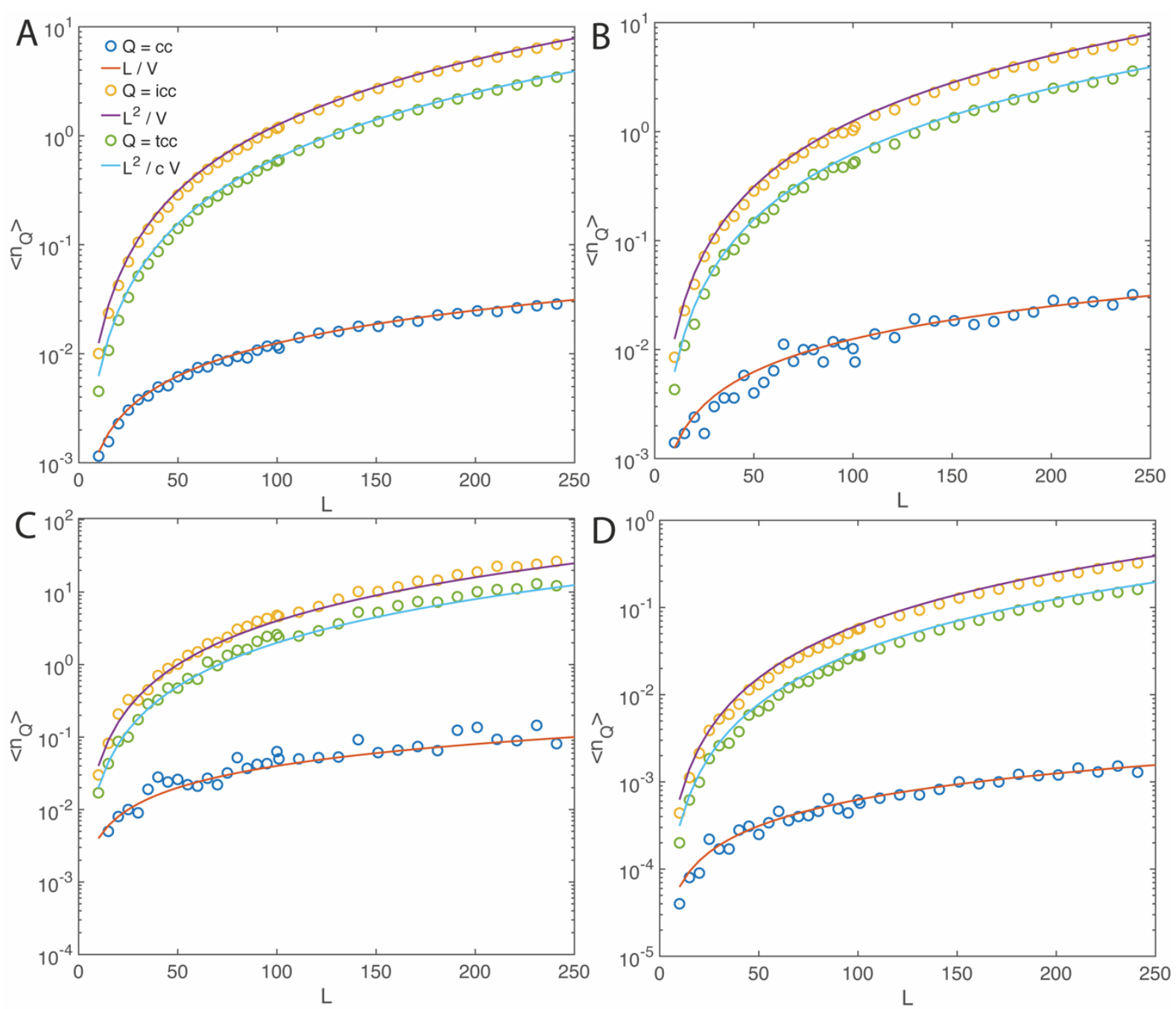

FIGURE 4. Variation of the average number of correct $\left(n_{c c}\right)$, incorrect $\left(n_{i c c}\right)$ and trap correct contacts $\left(n_{t c c}\right)$ with respect to the length $L$ of SARWs and dimensionality. Average number of various contacts were obtained over $10^{5}$ SARW trajectories. Hollow circles are the stochastic simulations results and solid lines are the predictions by Eqs. 2. Irrespective of the dimensionality and shape of the lattice box where the volume of the monomer unit is $v=1$, the expression given in Eqs. 2 are valid. A. Three dimensional SARW confined in volume $V=$ $20^{3}$, length was iterated in $L=(10,250)$ and $c=2$. B. Three dimensional SARW confined inside a box with base area $=10^{2}(10$ by 10$)$ and length $=80$ so that $V=8000$. The length of SARW is iterated inside $L=(10,250)$ with complexity $c=2$. $C$. two dimensional SARW confined in $V=$ $50^{2}$, and the length of SARW was iterated inside $L=(10,250)$ and $c=2$. D. Four dimensional SARW confined in $V=20^{4}, L=(10,250)$ and $c=2$. 
Lattice model on DNA hybridization
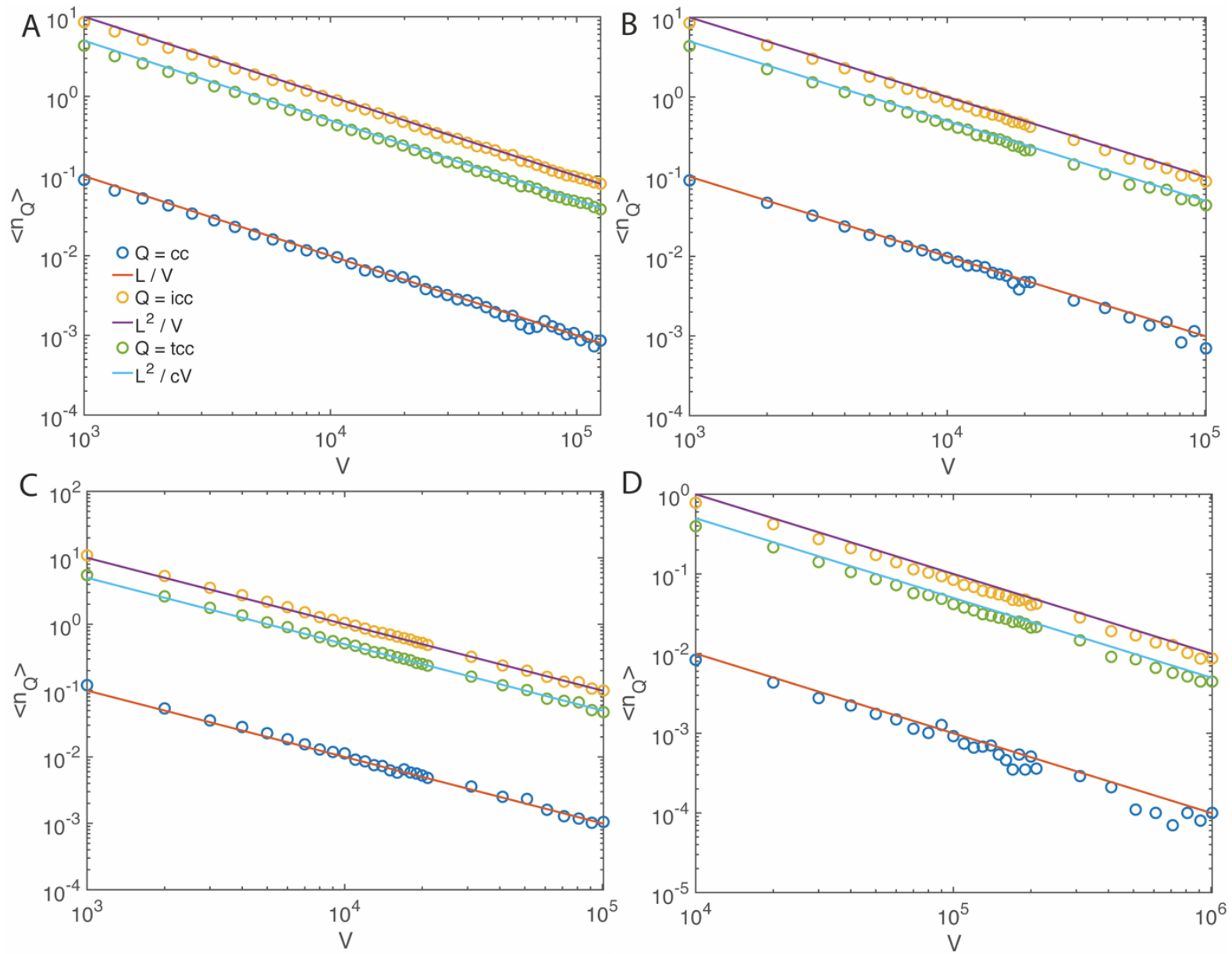

FIGURE 5. Variation of the average number of correct $\left(n_{c c}\right)$, incorrect $\left(n_{\text {icc }}\right)$ and trap correct contacts $\left(n_{t c c}\right)$ with respect to change in confinement volume of SARW and dimensionality. Average number of various types of contacts were obtained over $10^{5}$ trajectories. Hollow circles are the stochastic simulations results and solid lines are the predictions by Eqs. 2. Irrespective of the dimensionality and shape of the lattice box where the volume of the monomer unit is $v=1$, the expressions given in Eqs. 2 are valid. A. three dimensional SARW confined in cubic box with volumes iterated inside $V=\left(10^{3}, 50^{3}\right)$, length of SARW was set as $L$ $=100$ and $c=2$. B. three dimensional SARW confined inside a box with base area $=10^{2}$ and length iterated from 10 to 1000 so that $V$ varies from $10^{3}$ to $10^{5}$. The length of SARW is $L=$ 100 with complexity $c=2$. C. two dimensional SARW confined box with base side $=100$ and length iterated from 10 to 1000 so that $V$ varies from $10^{3}$ to $10^{5}, L=100$ and $C=2$. D. Four dimensional SARW confined in a box with base $10^{3}(10 \times 10 \times 10)$ and other side iterated from 10 to 1000 so that the volume $V$ varies from $10^{4}$ to $10^{6}$, length of SARW was set as $L=100$ and the complexity was set as $c=2$. 
Lattice model on DNA hybridization

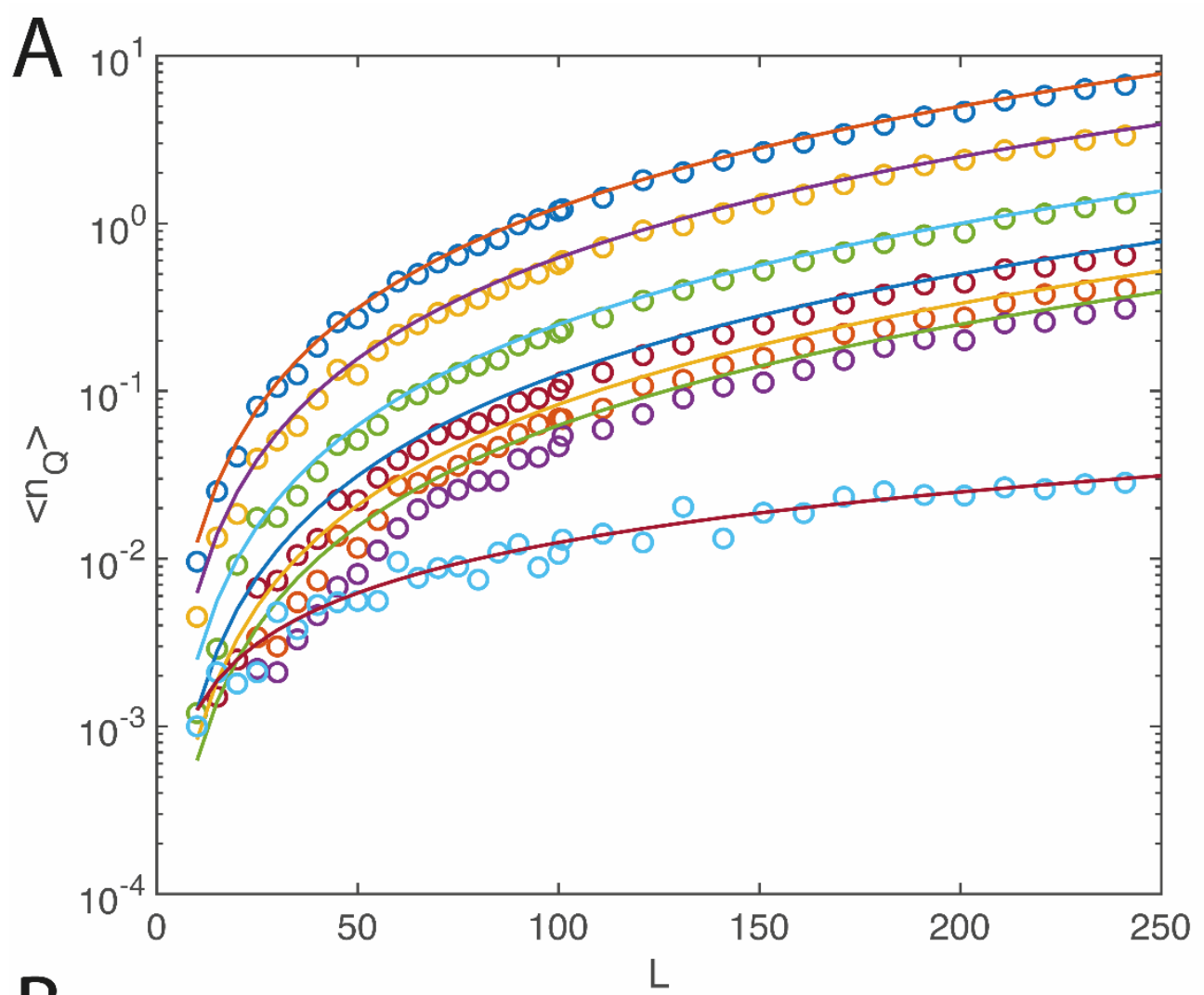

$O C=L, Q=i c c$

$-\mathrm{L}^{2} / \mathrm{V}$

$O \mathrm{C}=2, \mathrm{Q}=\mathrm{tcc}$

$-\mathrm{L}^{2} / 2 \mathrm{~V}$

$O C=5, Q=$ tcc

$-\mathrm{L}^{2} / 5 \mathrm{~V}$

$O C=10, Q=t c c$

$-\mathrm{L}^{2} / 10 \mathrm{~V}$

$O C=15, Q=$ tcc

$-\mathrm{L}^{2} / 15$

$O C=20, Q=$ tcc

$-\mathrm{L}^{2} / 20 \mathrm{~V}$

$O C=L, Q=C C$

$-\mathrm{L} / \mathrm{V}$

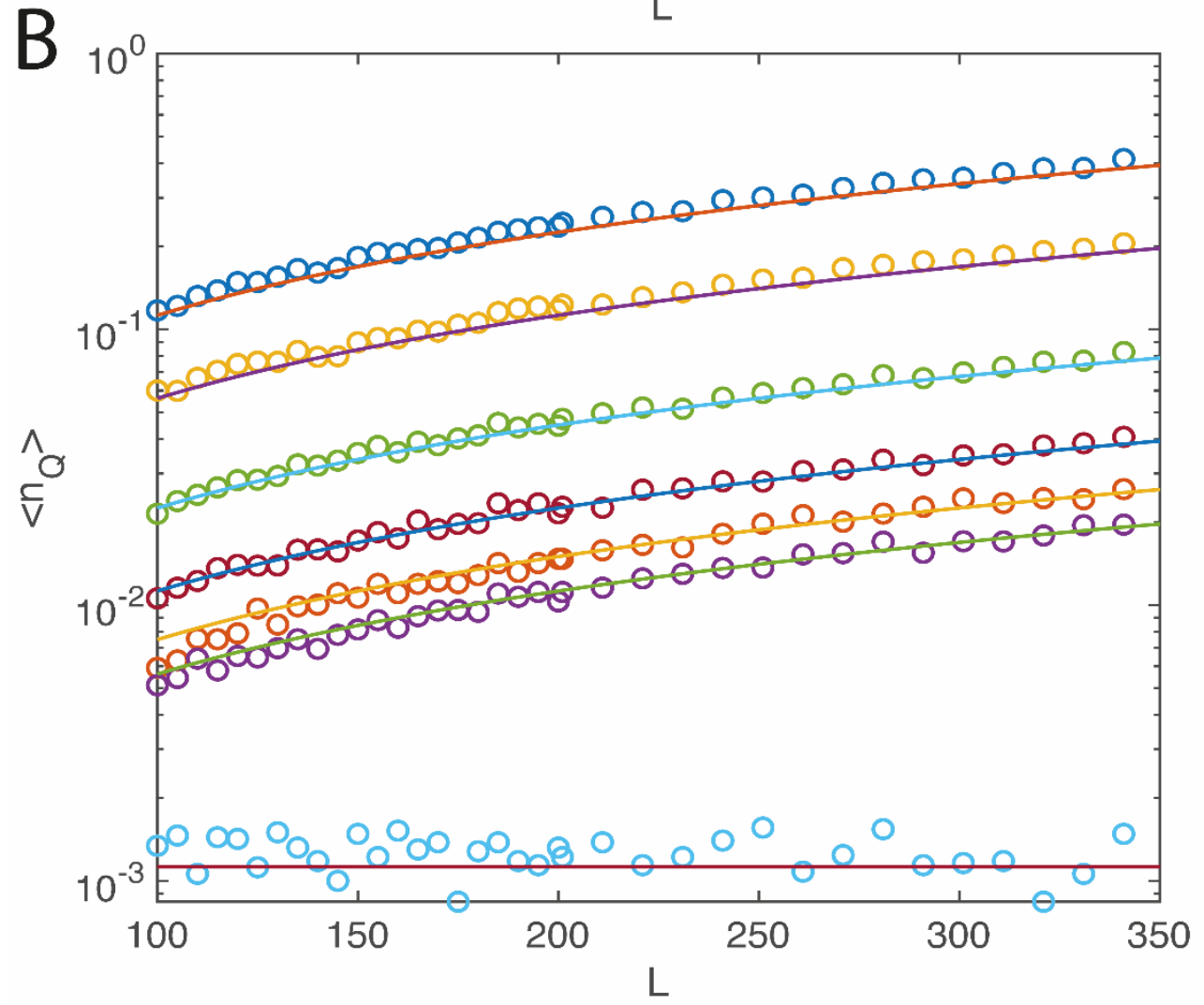

$\mathrm{O} c=\mathrm{L}, \mathrm{Q}=\mathrm{icc}$

- Lu /V

$O \mathrm{c}=2, \mathrm{Q}=\mathrm{tcc}$

- $\mathrm{Lu} / 2 \mathrm{~V}$

$\mathrm{O} c=5, \mathrm{Q}=\mathrm{tcc}$

$-\mathrm{Lu} / 5 \mathrm{~V}$

$O c=10, Q=t c c$

- Lu / 10V

$\mathrm{O} C=15, \mathrm{Q}=\mathrm{tcc}$

- Lu / 15V

$O c=20, Q=t c c$

- Lu / $20 \mathrm{~V}$

$O \mathrm{C}=\mathrm{L}, \mathrm{Q}=\mathrm{CC}$

$-\mathrm{u} / \mathrm{V}$

FIGURE 6. Variation of the average number of correct $\left(n_{c c}\right)$, incorrect $\left(n_{i c c}\right)$ and trap correct contacts $\left(n_{t c c}\right)$ with respect to changes in length $L$ and complexity c of SARWs. Average number of various types of contacts were obtained over $10^{5}$ trajectories Hollow circles are the stochastic simulations results and solid lines are the predictions by Eqs. 3. Here the volume of the monomer unit is set as $v=1$. A. the volume is fixed at $V=20^{3}$. B. the template strand 
length is fixed at $L=100$ and the length of probe is fixed at $u=10$. The probe was set to form duplex with the template at position stretch 40 to 50 . This is the recognition stretch of the probe ssDNA. Both these strands are embedded inside the volume $V=20^{3}$.
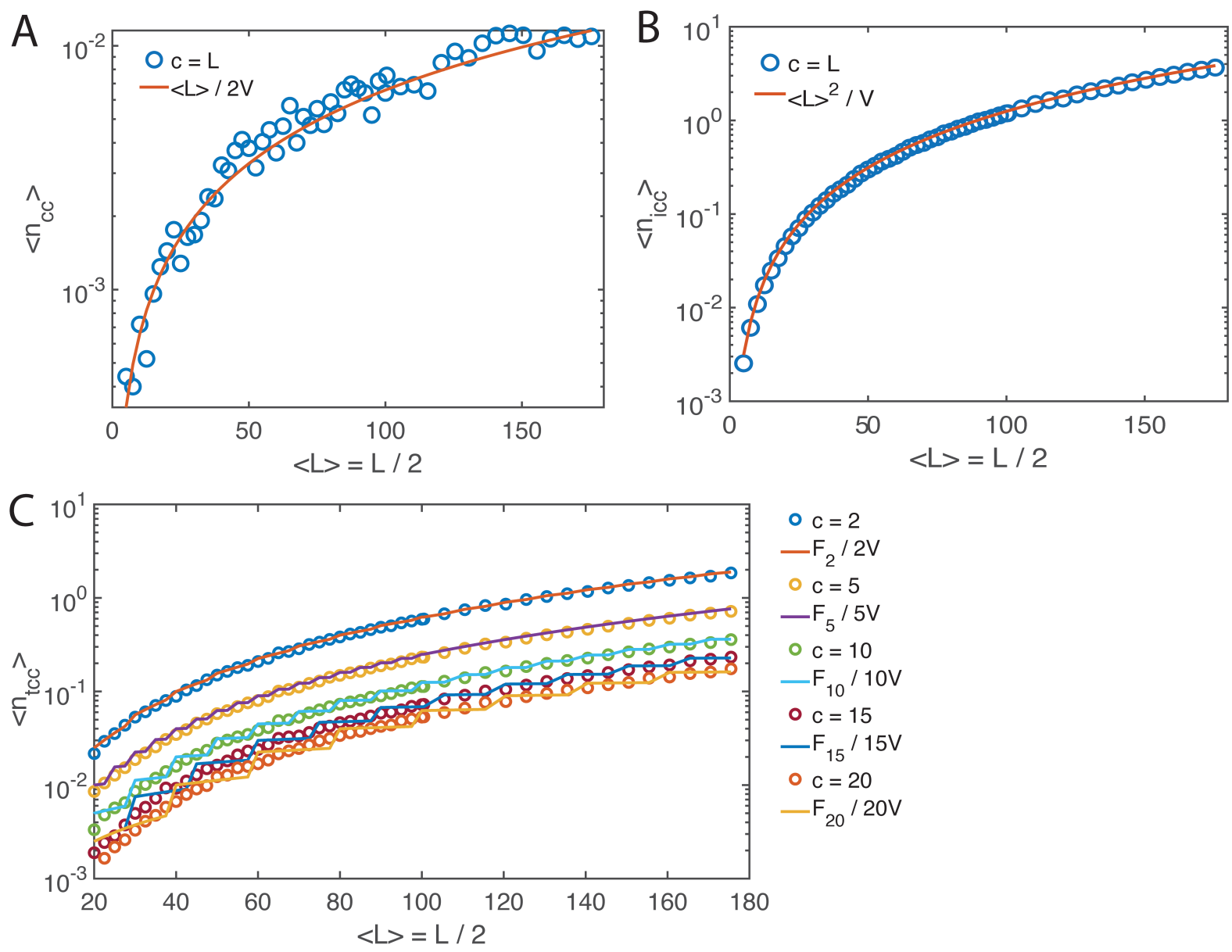

FIGURE 7. Variation of the average number of correct $\left(n_{c c}\right)$, incorrect $\left(n_{\text {icc }}\right)$ and trap correct contacts $\left(n_{t c c}\right)$ with respect to changes in the randomized lengths and complexity $c$ of (cSSDNAs) SARWs. Average number of various types of contacts were obtained over $10^{5}$ SARW trajectories. Hollow circles are the stochastic simulation results and solid lines are the predictions by Eqs. 4. Here the volume is set at $V=20^{3}$ and the length of SARW was chosen randomly within $(0, L)$ with equal probabilities so that the average length is $\angle L\rangle=L / 2$. Here $L$ was iterated inside $(10,360)$ so that the average length $\langle L>$ will vary from 5 to 180 . Results suggest that the probability of finding the correct contact upon collision is $1 / L$ rather than 1 $/<L>$ which leads to $\left\langle n_{c c}\right\rangle=L / 4 V$. A. $\left\langle n_{c c}\right\rangle$. B. $\left\langle n_{i c c}>\right.$. C. $\left\langle n_{t c c}\right\rangle$. When the sequence complexity is close to the sequence length, then one finds the approximation $\lim _{c \rightarrow\langle L\rangle}\left\langle n_{t c c}\right\rangle \cong \frac{v\left(\langle\bar{L}\rangle^{2}+\langle l\rangle^{2}\right)}{c V}$ where $\langle\bar{L}\rangle=c\left\lfloor\frac{\langle L\rangle}{c}\right\rfloor$ is the length of DNA containing full repeats and $\langle l\rangle=\langle L\rangle-c\left\lfloor\frac{\langle L\rangle}{c}\right\rfloor$ is the length of DNA left over with partial repeat. Here $v=1$ and $F_{c}=\langle\bar{L}\rangle^{2}+\langle l\rangle^{2}$. One also should note that Eqs. 4 will be valid for the repetitive DNA sequences only when $(L / c) \gg 1$. 

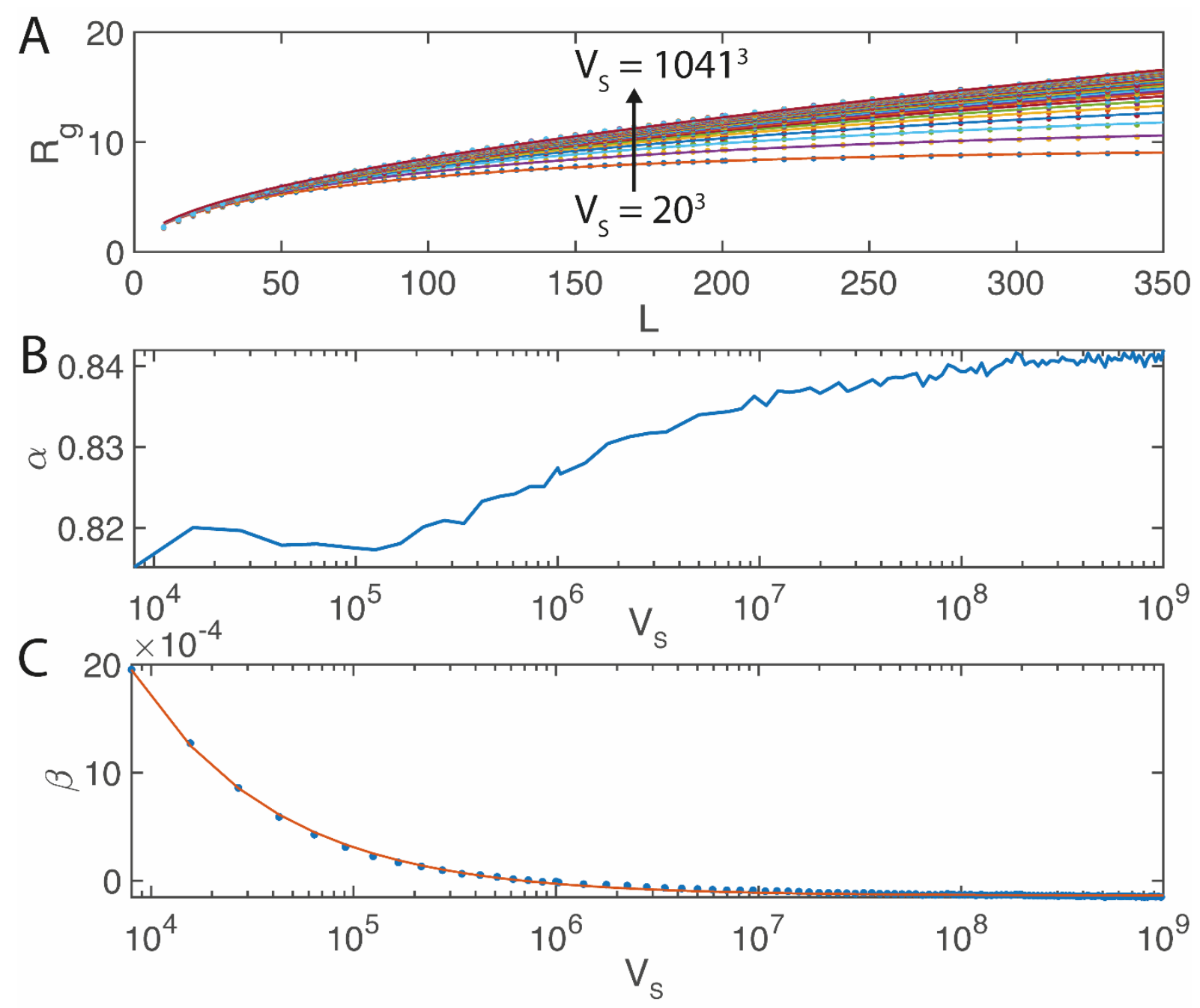

FIGURE 8. Variation of the average radius of gyration $R_{g}$ of SARWs with respect to changes in the length $L$ and the confinement volume $V_{S}$ of SARW. The radius of gyration of a chain molecule was calculated as $R_{g}=\left\langle\left|\sqrt{\frac{\sum_{i, j=1}^{L}\left(\vec{w}_{i}-\vec{w}_{j}\right)^{2}}{2 L^{2}}}\right|\right\rangle$ where $\vec{w}_{i}$ is the vector coordinates $(\mathrm{X}, \mathrm{Y}$, Z) of the $i^{t h}$ monomer and $L$ is the total length. Average of $R_{g}$ was obtained over $10^{5}$ numbers of SARW trajectories. Here filled circles in $A$ and $C$ are the numerical results and solid lines are the nonlinear least square (NLS) fittings. A. NLS fits to function $R_{g} \cong \alpha \sqrt{L}(1+\beta L)^{-1}$ with respect to $L$ at various $V_{s}$ values revealed the parameter $\alpha$ that does not change much with $V_{s}$ as given in $\mathbf{B}$ and $\beta=\left(\varepsilon\left(\frac{v}{V_{S}}\right)^{\delta}+\sigma\right)$ as given in $\mathbf{C}$ where $\varepsilon=0.5 \pm 0.04, \delta=0.61 \pm 0.1$ and $\sigma=-10^{-4} \pm 10^{-5}$. These values were obtained with $95 \%$ confidence level. Here the monomer volume is set to $v=1$ cubic unit. 
A

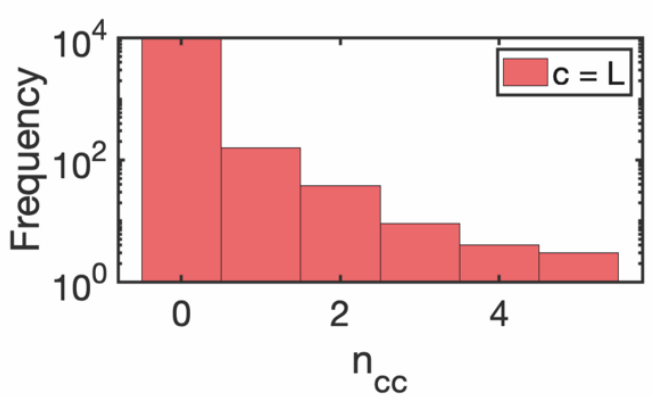

C

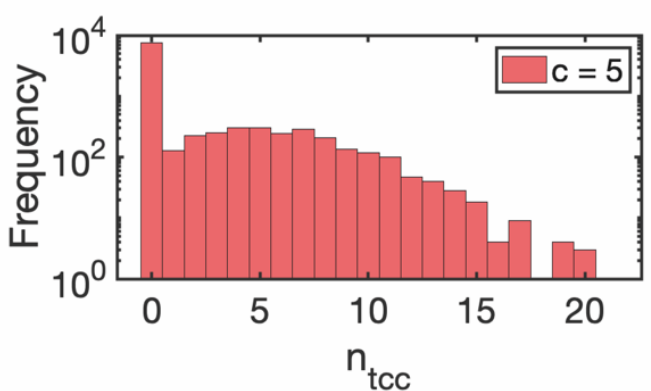

$\mathrm{E}$

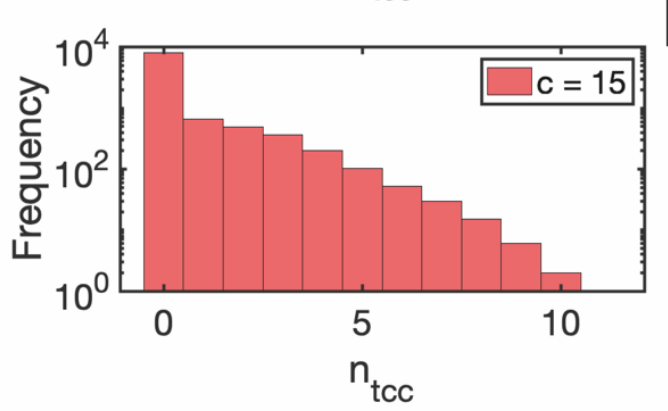

B

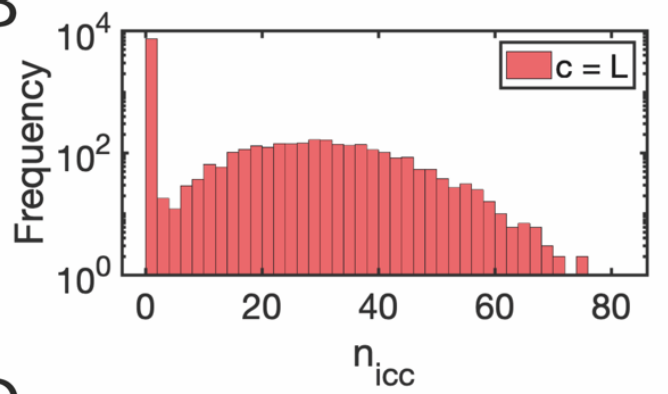

D

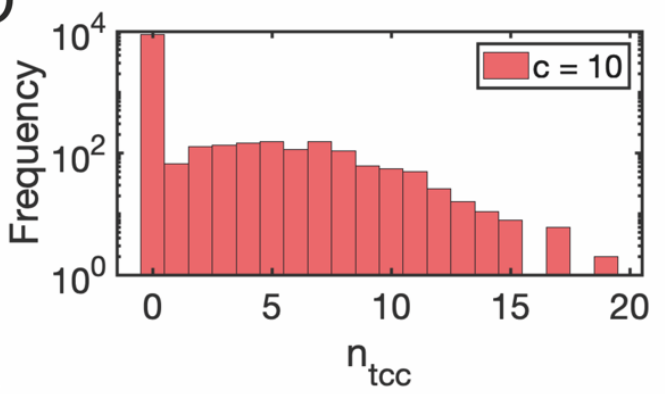

F

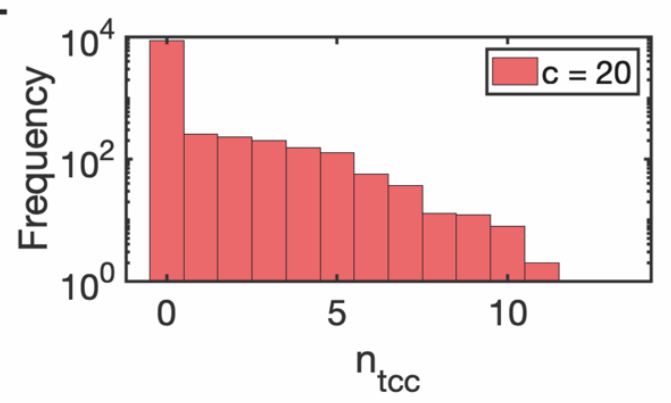

FIGURE 9. Distributions of the number of correct $\left(n_{c c}\right)$, incorrect $\left(n_{i c c}\right)$ and trap correct contacts $\left(n_{t c c}\right)$ at various sequence complexity $c$ values. Here volume of the lattice cube is set as $V=$ $20^{3}$, the length of SARW is fixed at $L=250$ and the sequence complexity $\mathrm{c}$ was varied as $\mathrm{c}=$ $(5,10,15,20,250)$. Histograms of various types of contacts were constructed over $10^{4}$ numbers of SARW trajectories. Clearly, all these distributions show bimodal type with zero spike that represents no contact cases. A-B. complexity is set to $c=250$. C. $c=5$. D. $c=10$. E. $c=15$. F. $c=20$. 
Lattice model on DNA hybridization

TABLE 1. List of symbols and definitions used in the main text

\begin{tabular}{|c|c|c|}
\hline Symbol & Definition & Remarks \\
\hline SARW & Self-avoiding random walk. & \\
\hline 1D, 3D & One-dimensional, three-dimensional. & \\
\hline dsDNA & Double stranded DNA. & \\
\hline ssDNA & Single stranded DNA. & \\
\hline c-ssDNA & $\begin{array}{l}\text { Complementary single stranded DNA, which can form } \\
\text { dsDNA. }\end{array}$ & \\
\hline cc, icc, tcc & Correct-contact, incorrect contact and trap correct contacts. & \\
\hline$I_{p}$ & $\begin{array}{l}=3.4 \times 10^{-10} \mathrm{~m}=1 \mathrm{bp} \text {, Distance between two consecutive } \\
\text { bases of } \mathrm{dsDNA} \text {. }\end{array}$ & $1 \mathrm{bp}$ \\
\hline$L, u$ & Length of the template and probe ssDNA polymers. & $\begin{array}{l}\text { Nucleotides, } \\
\text { nt }\end{array}$ \\
\hline$v$ & Volume of a nucleotide monomer. & $b p^{3}$ \\
\hline V & $\begin{array}{l}\text { Reaction volume which is equal to the sphere with radius } \\
\text { equal to the sum of the radii of gyration of c-ssDNAs. }\end{array}$ & $\mathrm{m}^{3}$ \\
\hline$v L$ & Volume of the ssDNA polymer of length $\mathrm{L}$. & $\mathrm{m}^{3}$ \\
\hline$c$ & $\begin{array}{l}\text { Complexity of DNA sequence. Number of unique bases } \\
\text { present in the given DNA sequence. }\end{array}$ & $\mathrm{nt}$ \\
\hline$<n_{c c}>$ & $\begin{array}{l}(=v L / V) \text {, average number of correct contacts between the } \\
\text { complementary ssDNA strands when they are confined } \\
\text { within a reaction volume } V \text {. }\end{array}$ & dimensionless \\
\hline$<n_{i c c}>$ & $\begin{array}{l}\left(=v L^{2} / V\right) \text {, average number of incorrect contacts formed } \\
\text { between the complementary ssDNA strands when they are } \\
\text { confined within a reaction volume } V \text {. }\end{array}$ & dimensionless \\
\hline$<n_{t c c}>$ & $\begin{array}{l}\left(=v L^{2} / c V\right) \text {, average number of trap correct contacts formed } \\
\text { between the complementary ssDNA strands with complexity } \\
c \text { when they are confined within a reaction volume } V \text {. }\end{array}$ & dimensionless \\
\hline$k_{t}$ & $\begin{array}{l}=k_{s m} \phi \text {, where } k_{s m} \text { is the maximum possible Smoluchowski } \\
\text { rate limit and } \phi \text { is the factor associated with the electrostatic } \\
\text { repulsions at the DNA-DNA interface. }\end{array}$ & (mol/lit/s) \\
\hline$k_{c c}$ & $\begin{array}{l}=k_{t}\left\langle n_{c c}\right\rangle \text {, is the number of correct contacts formed upon } \\
\text { interpenetration of c-ssDNAs. }\end{array}$ & (mol/lit/s) \\
\hline$k_{\text {icc }}$ & $\begin{array}{l}=k_{t}\left\langle n_{i c c}\right\rangle \text {, is the number of incorrect contacts formed upon } \\
\text { interpenetration of c-ssDNAs. }\end{array}$ & (mol/lit/s) \\
\hline$k_{t c c}$ & $\begin{array}{l}=k_{t}\left\langle n_{t c c}\right\rangle \text {, is the number of trap correct contacts formed } \\
\text { upon interpenetration of repetitive c-ssDNAs. }\end{array}$ & (mol/lit/s) \\
\hline$k_{N}$ & Nucleation rate constant that directly proportional to $k_{c c}$. & (mol/lit/s) \\
\hline$k_{z}$ & Zipping rate constant. & $1 / \mathrm{s}$ \\
\hline$M_{1}, M_{2}$ & Concentrations of c-ssDNA strand 1 and 2. & (mol/lit) \\
\hline$M_{D}$ & Concentration of duplex form DNA. & (mol/lit) \\
\hline$W$ & Concentration of nucleus DNA. & (mol/lit) \\
\hline$Y$ & Concentration of incorrect contacts. & (mol/lit) \\
\hline$N$ & Size of the nucleus. & nt \\
\hline$R_{g}$ & Average radius of gyration of c-ssDNA or SARW. & $\mathrm{bp}$ \\
\hline
\end{tabular}


bioRxiv preprint doi: https://doi.org/10.1101/2021.12.22.473940; this version posted December $23,2021$. The copyright holder for this preprint (which was not certified by peer review) is the author/funder, who has granted bioRxiv a license to display the preprint in perpetuity. It is made available under aCC-BY-ND 4.0 International license.

Lattice model on DNA hybridization

\begin{tabular}{|c|l|l|}
\hline$q, q_{t}$ & $\begin{array}{l}\text { Slithering lengths associated with the complementary } \\
\text { ssDNAs with icc and tcc. }\end{array}$ & $\mathrm{nt}$ \\
\hline$k_{r}, k_{r t}$ & $\begin{array}{l}\text { Dissociation rates associated with c-ssDNAs having icc and } \\
\text { tcc respectively. }\end{array}$ & $1 /$ second \\
\hline$k_{R}$ & $\begin{array}{l}\text { Overall renaturation rate. } k_{R Q} \text { where Q }=\mathrm{I}, \mathrm{II}, \text { III and IV } \\
\text { correspond to the respective renaturation Schemes I, II, III } \\
\text { and IV. }\end{array}$ & (mol/lit/s) \\
\hline$k_{+} k_{-}$ & Microscopic forward and reverse zipping rate constants. & $\mathrm{nt} / \mathrm{second}$ \\
\hline
\end{tabular}

